\title{
INTERPOLATION SETS AND THE SIZE OF QUOTIENTS OF FUNCTION SPACES ON A LOCALLY COMPACT GROUP
}

\author{
M. FILALI AND J. GALINDO
}

\begin{abstract}
We devise a fairly general method for estimating the size of quotients between algebras of functions on a locally compact group. This method is based on the concept of interpolation set we introduced and studied recently and unifies the approaches followed by many authors to obtain particular cases.

We find in this way that there is a linear isometric copy of $\ell_{\infty}(\kappa)$ in each of the following quotient spaces:

- $\mathcal{W A P}_{0}(G) / C_{0}(G)$ whenever $G$ contains a subset $X$ that is an $E$ set (see the definition in the paper) and $\kappa=\kappa(X)$ is the minimal number of compact sets required to cover $X$. In particular, $\kappa=$ $\kappa(G)$ when $G$ is a $S I N$-group.

- $\mathcal{W} \mathcal{A} \mathcal{P}(G) / \mathcal{B}(G)$, when $G$ is any locally compact group and $\kappa=$ $\kappa(Z(G))$ and $Z(G)$ is the centre of $G$, or when $G$ is either an $I N$-group or a nilpotent group, and $\kappa=\kappa(G)$.

- $\mathcal{W} \mathcal{A} \mathcal{P}_{0}(G) / \mathcal{B}_{0}(G)$, when $G$ and $\kappa$ are as in the foregoing item.

- $\mathcal{E}(G) / \mathcal{L U C}(G)$, when $G$ is any locally compact group that is neither compact nor discrete and $\kappa=\kappa(G)$.
\end{abstract}

\section{INTRODUCTION}

The main focus throughout the paper will be on $C^{*}$-algebras of functions on a locally compact group $G$ with identity $e$. If $\ell_{\infty}(G)$ denotes the $C^{*}$ algebra of bounded, scalar-valued functions on $G$ with the supremum norm, our concern will be with the following subalgebras of $\ell_{\infty}(G)$ : the algebra $\mathcal{E B}(G)$ of continuous bounded functions, the algebra $\mathcal{L} \mathcal{U}(G)$ of bounded right uniformly continuous functions, the algebra $\mathcal{W} \mathcal{A P}(G)$ of weakly almost periodic functions, the Fourier-Stieltjes algebra $B(G)$, the uniform closure of $B(G)$ denoted by $\mathcal{B}(G)$ and best known as the Eberlein algebra, the algebra $\mathcal{A P}(G)$ of almost periodic functions, and the algebra $C_{0}(G) \oplus \mathbb{C} 1$, where $C_{0}(G)$ consists of the functions in $\mathcal{E} \mathcal{B}(G)$ vanishing at infinity.

The spectra of these algebras $\mathcal{A}(G)$ define some of the best-known semigroup compactifications in the sense of [5]. These are compact right (or left)

Date: October 15, 2014.

2010 Mathematics Subject Classification. Primary 22D15; Secondary 43A46, 43A15, $43 \mathrm{~A} 60$.

Key words and phrases. almost periodic functions, Fourier-Stieltjes algebra, weakly almost periodic, semigroup compactification, almost periodic compactification, almost periodic compactification, interpolation sets.

Research of the second named author supported by the Spanish Ministry of Science (including FEDER funds), grant MTM2008-04599/MTM and Fundació Caixa CastellóBancaixa, grant P1.1B2008-26. 
topological semigroups $G^{\mathcal{A}}$ having a dense, continuous, homomorphic copy of $G$ contained in their topological centres (i.e, the map $x \mapsto s x \quad(x \mapsto x s)$ : $G^{\mathcal{A}} \rightarrow G^{\mathcal{A}}$ is continuous for each $s \in G$.) For instance, the compactification $G^{\mathcal{L U}}$ is the spectrum of $\mathcal{L U} \mathrm{C}(G)$, and is usually referred to as the $\mathcal{L U} \mathcal{U}(G)$ or $\mathcal{L} \mathcal{C}(G)$-compactification of $G$. It is the largest semigroup compactification in the sense that any other semigroup compactification is a quotient of $G^{\mathcal{L} U \mathcal{C}}$. When $G$ is discrete, $G^{\mathcal{L U C}}$ and the Stone-Čech compactification $\beta G$ are the same. The $\mathcal{W} \mathcal{A P}$-compactification $G^{\mathcal{W A P}}$ is the spectrum of $\mathcal{W} \mathcal{A P}(G)$; it is the largest semitopological semigroup compactification. The Bohr or $\mathcal{A P}$-compactifiaction is the spectrum of $\mathcal{A P}(G)$ and is the largest topological (semi)group compactification.

The Banach duals of these $C^{*}$-algebras can also be made into Banach algebras with a convolution type product extending in most cases that of the group algebra $L^{1}(G)$. We may recall that $L^{\infty}(G)$ is the Banach dual of the group algebra $L^{1}(G)$ and consists of all scalar-valued functions which are measurable and essentially bounded with respect to the Haar measure; two functions are identified if they coincide on a locally null set, and the norm is given by the essential supremum norm. We may also recall that the product making $L^{\infty}(G)^{*}$ into a Banach algebra is the first (or the second) Arens product on the second dual space $L^{1}(G)^{* *}$ of the group algebra, and that $\mathcal{L U C}(G)^{*}$ may be seen as a quotient Banach algebra of $L^{1}(G)^{* *}$. For more details, see for instance [17]. These two Banach algebras have been studied extensively in recent years. Particular attention has been given to properties related to Arens regularity of the group algebra $L^{1}(G)$ and to the topological centres of $G^{\mathcal{L U C}}, \mathcal{L U C}(G)^{*}$ and $L^{1}(G)^{* *}$. For the latest, see [8] and the references therein.

The definitions of all these function algebras will be given in the next section. But for the moment the following diagram summarizes already the inclusion relationships known to hold among these algebras. See [13, page 143] for the first inclusion; [13, Lemma 2.1] for the first equality; [42] or [5, Theorem 4.3.13] for the second equality; [5, Corollary 4.4.11] or [9] for the third inclusion; the rest is easy to check.

$$
\begin{aligned}
C_{0}(G) \oplus \mathcal{A P}(G) & \subseteq \mathcal{B}(G)=\mathcal{A P}(G) \oplus \mathcal{B}_{0}(G) \subseteq \mathcal{W} \mathcal{A P}(G)=\mathcal{A P}(G) \oplus \mathcal{W} \mathcal{A P} \mathcal{P}_{0}(G) \\
& \subseteq \mathcal{L U} \mathcal{C}(G) \cap \mathcal{R U C}(G) \subseteq \mathcal{L U} \mathcal{C}(G) \subseteq \mathcal{C B}(G) \\
& \subseteq L^{\infty}(G) .
\end{aligned}
$$

When $G$ is finite, the diagram is trivial. When $G$ is infinite and compact, the diagram reduces to $\mathcal{C} \mathcal{B}(G) \subseteq L^{\infty}(G)$.

The task of comparing these algebras and estimating the sizes of the quotients formed among them has already been taken by many authors. We now give a brief review of what is known in this respect.

In the review below as well as in our study of quotients between the above algebras the compact covering number will appear at several points. We recall that the compact covering number of a topological space $X$ is the smallest cardinal number $\kappa(X)$ of compact subsets of $X$ required to cover $X$.

Comparing $L^{\infty}(G)$ With its subspaces. Already in 1961, Civin and Yood proved in their seminal paper [15] that the quotient space $L^{\infty}(G) / \mathcal{C} \mathcal{B}(G)$ 
is infinite-dimensional for any non-discrete locally compact Abelian group and deduced that the radical of the Banach algebra $L^{\infty}(G)^{*}$ (with one of the Arens products as a product) is also infinite-dimensional.

This idea was pushed further by Gulick in [34, Lemma 5.2] when $G$ is Abelian, and proved that the quotient $L^{\infty}(G) / \mathcal{C B}(G)$ is even non-separable and so is the radical of $L^{\infty}(G)^{*}$. Then Granirer proved in [32] the same results for any non-discrete locally compact group.

A decade later, Young produced, for any infinite locally compact group $G$, a function in $L^{\infty}(G)$ which is not in $\mathcal{W} \mathcal{A P}(G)$, proving the non-Arens regularity of the group algebra $L^{1}(G)$ for any such a group, see [54].

There was also $[6$, Theorem 4.2] where the quotient $\mathcal{L} \mathcal{U}(G) / \mathcal{W} \mathcal{A P}(G)$ was seen to contain a linear isometric copy of $\ell_{\infty}(\kappa)$, where $\kappa$ is the compact covering of $G$. A fortiori, the quotient $L^{\infty}(G) / \mathcal{W} \mathcal{A P}(G)$ contains the same copy, a fact that was used in [6, Theorem 4.4] to deduce that the group algebra is even extremely non-Arens regular in the sense of Granirer, whenever $\kappa$ is larger than or equal to the minimal cardinal $\chi(G)$ of a basis of neighbourhoods at the identity.

It was also proved in $[6$, Section 4$]$ that the quotient $L^{\infty}(G) / \mathcal{C B}(G)$ always contains a linear isometric copy of $\ell_{\infty}$, yielding extreme non-Arens regularity for the group algebra of compact metrizable groups. Due to a result by Rosenthal proved in [48, Proposition 4.7, Theorem 4.8], larger copies of $\ell_{\infty}$ cannot be expected in $L^{\infty}(G)$ when $G$ is compact. The question on extreme non-Arens regularity of the group algebra was recently settled by the authors of the present paper using a technique inspired by Theorem 2.11. We actually find in [25] that, for any compact group $G, L^{\infty}(G) / \mathcal{C B}(G)$ contains a copy of $L^{\infty}(G)$. This fact together with [6, Theorem 4.4] gives that $L^{1}(G)$ is extremely non-Arens regular for any infinite locally compact group.

Comparing $\mathcal{C} B(G)$ with its subspaces. In 1966, Comfort and Ross [16, Theorem 4.1] compared the spaces $\mathcal{C} \mathcal{B}(G)$ and $\mathcal{A P}(G)$ for an arbitrary topological group, and proved that they are equal if and only if $G$ is pseudocompact (i.e., every continuous scalar-valued function on $G$ is bounded). In 1970, Burckel showed in [9] that $\mathcal{C B}(G)$ and $\mathcal{W} \mathcal{A P}(G)$ are equal if and only if $G$ is compact. In [3], Baker and Butcher compared $\mathcal{E} \mathcal{B}(G)$ and $\mathcal{L U} \mathcal{C}(G)$ for locally compact groups, and proved that these two spaces are equal if and only if $G$ is either discrete or compact. This result was extended recently by Filali and Vedenjuoksu in [28, Theorem 4.3] to all topological groups which are not $P$-groups. The author deduced in [28] that if $G$ is a topological group which is not a $P$-group, then $\mathcal{C B}(G)=\mathcal{L U} \mathcal{C}(G)$ if and only if $G$ is pseudocompact. In [20], Dzinotyiweyi showed that the quotient $\mathcal{C B}(G) / \mathcal{L U C}(G)$ is non-separable if $G$ is a non-compact, non-discrete, locally compact group. This theorem was generalized in [6, Theorem 3.1] and [7, Theorem 4.1], where $\mathcal{C B}(G) / \mathcal{L U C}(G))$ was seen to contain in fact a linear isometric copy of $\ell_{\infty}$ whenever $G$ is a non-precompact topological group which is not a $P$-group. So this theorem improved actually also Dzinotyiweyi's result for locally compact groups. For non-discrete, $P$-groups, the quotient $\mathcal{C B}(G) / \mathcal{L U C}(G)$ was seen to be trivial in the case when for instance 
$G$ is a Lindelöf $P$-group (see [28, Theorem 5.1]), but may also contain a linear isometric copy of $\ell_{\infty}$ for some other $P$-groups (see [6, Theorem 3.3]). In [7, Theorem 3.1], using a technique due to Alas (see [1]), the quotient space $\mathcal{C} \mathcal{B}(G) / \mathcal{L U} \mathcal{C}(G)$ was also seen to contain a linear isometric copy of $\ell_{\infty}$ whenever $G$ is a non-SIN topological group.

In the locally compact situation, our answer in the present paper is precise and definite. We prove, in Section 5, that there is a linear isometric copy of $\ell_{\infty}(\kappa)$ in $\mathcal{C} \mathcal{B}(G) / \mathcal{L U} \mathcal{C}(G)$, where as before $\kappa$ is the compact covering $G$, if and only if $G$ is a neither compact nor discrete. This leads again to a linear isometric copy of $\ell_{\infty}(\kappa)$ into the quotient $L^{\infty}(G) / \mathcal{W} \mathcal{A P}(G)$, and of course may be used to deduce again the extreme non-Arens regularity of of $L^{1}(G)$ when $\kappa(G) \geq \chi(G) \geq \omega$ as in [6, Theorem 4.4].

Comparing $\mathcal{L U} \mathcal{U}(G)$ With $\mathcal{W} \mathcal{A P}(G)$. In 1972 , Granirer showed that $\mathcal{L U C}(G)=\mathcal{W} \mathcal{A P}(G)$ if and only if $\mathrm{G}$ is compact [31].

It is not difficult to check that $G^{\mathcal{L U}}$ is a semitopological semigroup (i.e., the topological centre of $G^{\mathcal{L U C}}$ is the whole of $G^{\mathcal{L U}}$ ) if and only if $\mathcal{L U C}(G)=$ $\mathcal{W} \mathcal{A P}(G)$. The same observation can be made also for $\mathcal{L U C}(G)^{*}$. This means that $G^{\mathcal{L U C}}$ or $\mathcal{L U C}(G)^{*}$ is a semitopological semigroup if and only if $G$ is compact, i.e., $G^{\mathcal{L U C}}=G$ is a compact group and $\mathcal{L U C}(G)^{*}$ coincides with the measure algebra $M(G)$.

More recently, Granirer's result was deduced by Lau and Pym in [40, Proposition 3.6] as a corollary of their main theorem on the topological centre of $G^{\mathcal{L L}}$ being $G$, and again by Lau and Ülger in [41, Corollary 3.8] as a corollary of the topological centre of $L^{1}(G)^{* *}$ being $L^{1}(G)[39]$.

Moreover, Granirer showed in the same paper that if $G$ is non-compact and amenable, then the quotient $\mathcal{L U} \mathcal{U}(G) / \mathcal{W} \mathcal{A P}(G)$ contains a linear isometric copy of $\ell_{\infty}$, and so it is not separable. This result was extended by Chou in [10] to E-groups (see below for definition), then by Dzinotyiweyi in [20] to all non-compact locally compact groups, and generalized by Bouziad and Filali in [6, Theorem 2.2] to all non-precompact topological groups. Moreover, as already mentioned above, this result was improved in [6, Theorem 4.2] when $G$ is a non-compact locally compact group, by having a copy of $\ell_{\infty}(\kappa)$ in the quotient $\mathcal{L U} \mathcal{C}(G) / \mathcal{W} \mathcal{A P}(G)$.

Comparing $\mathcal{W} \mathcal{A} \mathcal{P}(G)$ With its subspaces. In the "regular" side of the inclusion diagram, when we compare $\mathcal{W} \mathcal{A} \mathcal{P}(G)$ with its subspaces, the situation is not simpler. It is true that the Fourier-Stieltjes algebra $B(G)$ may be dense in $\mathcal{W} \mathcal{A P}(G)$ (i.e., $\mathcal{W} \mathcal{A} \mathcal{P}(G)=\mathcal{B}(G)$ ), as in the case of minimally weakly almost periodic groups studied by Veech, Chou and Ruppert, see [53], [12] and [51]. For these groups, $\mathcal{W} \mathcal{A P}(G)=\mathcal{A P}(G) \oplus C_{0}(G)$. However, if $G$ is a non-compact group, then $B(G)$ is far from being dense in $\mathcal{W} \mathcal{A P}(G)$ in general as it shall soon be explained.

When comparing $\mathcal{W} \mathcal{A P}(G)$ with $\mathcal{A P}(G)$ and $C_{0}(G)$, we may recall first that $\mathcal{W} \mathcal{A P}(G)=\mathcal{A P}(G) \oplus \mathcal{W} \mathcal{A} \mathcal{P}_{0}(G)$. Burckel proved in [9] that $C_{0}(G) \subsetneq$ $\mathcal{W} \mathcal{A} \mathcal{P}_{0}(G)$ when $G$ is an Abelian, non-compact, locally compact group. In [10], Chou considered $E$-groups and proved that the quotient $\mathcal{W} \mathcal{A} \mathcal{P}_{0}(G) / C_{0}(G)$ contains a linear isometric copy of $\ell_{\infty}$. In Section 4 , we improve this result by showing that $\ell_{\infty}$ may be replaced by an isometric copy of the larger space 
$\ell_{\infty}(\kappa(E))$ in each of the quotient space $\mathcal{W} \mathcal{A} \mathcal{P}_{0}(G) / C_{0}(G)$, where $\kappa(E)$ is the compact covering of the $E$-set contained in $G$. So when $G$ is an $S I N$-group, these quotients contain a copy of $\ell_{\infty}(\kappa(G))$. For the same class of groups, we prove also that the quotient $\mathcal{W} \mathcal{A P}(G) /\left(\mathcal{A P}(G) \oplus C_{0}(G)\right)$ is non-separable.

In Section 5, we deal with the non-compact, $I N$-groups, and with noncompact nilpotent groups. In this class of groups, the results of the previous section shall be considerably improved. Rudin proved in [49] that $\mathcal{B}(G) \subsetneq$ $\mathcal{W} \mathcal{A P}(G)$ if $G$ is a locally compact Abelian group and contains a closed discrete subgroup which is not of bounded order. This was followed by [47], where Ramirez extended Rudin's result to any non-compact, locally compact, Abelian group. Then in [13], Chou extended and strengthened the theorem to all non-compact $I N$-groups and nilpotent groups by showing that the quotient $\mathcal{W} \mathcal{A P}(G) / \mathcal{B}(G)$ contains a linear isometric copy of $\ell_{\infty}$.

We shall strengthen Chou's result in Section 5 by showing that, in these cases, there is in fact a linear isometric copy of $\ell_{\infty}(\kappa)$ in the quotient spaces $\mathcal{W} \mathcal{A P}(G) / \mathcal{B}(G), \mathcal{W} \mathcal{A P}(G) /\left(\mathcal{A P}(G) \oplus C_{0}(G)\right)$ and $\mathcal{W} \mathcal{A} \mathcal{P}_{0}(G) / \mathcal{B}_{0}(G)$, where $\kappa$ is as before the compact covering of $G$. Our method of proof also shows that $\mathcal{W} \mathcal{A P}(G) / \mathcal{B}(G)$ always contains a copy of $\ell_{\infty}(\kappa(Z(G)))$.

It is worthwhile to note that all this confirms an observation made in $[5$, page 216], and gives indeed an indication on the size and complexity of the $\mathcal{W} \mathcal{A P}$-compactification $G^{\mathcal{W A P}}$ and the Banach algebra $\mathcal{W} \mathcal{A P}(G)^{*}$.

OutLine. The underlying structure in many of the proofs that estimate the size of $\mathcal{A}_{2}(G) / \mathcal{A}_{1}(G)$ for $C^{*}$-subalgebras $\mathcal{A}_{1}(G) \subseteq \mathcal{A}_{2}(G)$ of $\ell_{\infty}(G)$, depends on the existence of sets of interpolation for $\mathcal{A}_{2}(G)$ that are not sets of interpolation for $\mathcal{A}_{1}(G)$ (see for instance [6], [7], [10], [13] or [20]). One of the main objectives of the present paper is to make that structure emerge in a clear fashion. A first, but essential, step towards this objective is to work with the right concept of interpolation sets. We will use here the general concept of interpolation set introduced in [24] that extends several related classical ones and show how to apply it in this setting. The resulting interpolation sets are characterized in [24] in term of topological group properties, thereby making them easier to manipulate. We finally illustrate the scope of our approach by studying some concrete cases. We shall in particular study under this light the following quotients: $\mathcal{W A P}_{0}(G)$ by $C_{0}(G)$ and $\mathcal{W} \mathcal{A P}(G)$ by $\mathcal{A P}(G) \oplus C_{0}(G)$ for $E$-groups, $\mathcal{W} \mathcal{A P}(G)$ by $\mathcal{B}(G), \mathcal{W} \mathcal{A P}(G)$ by $\mathcal{A P}(G) \oplus C_{0}(G)$ and $\mathcal{W A P}_{0}(G)$ by $\mathcal{B}_{0}(G)$ for $I N$-groups and nilpotent groups, $\mathcal{C} \mathcal{B}(G)$ by $\mathcal{L U}(G)$ for locally compact groups.

1.1. The function algebras. We start by recalling the definitions of the function algebras we are interested in, for more details the reader is directed for example to [5].

Let $G$ be a topological group. For each function $f$ defined on $G$, the left translate $f_{s}$ of $f$ by $s \in G$ is defined on $G$ by $f_{s}(t)=f(s t)$. For each $s \in G$, the left translation operator $L_{s}: \ell_{\infty}(G) \rightarrow \ell_{\infty}(G)$ is defined as $L_{s}(f)=f_{s}$. The supremum norm of an element $f \in \ell_{\infty}(G)$ will be denoted as $\|f\|_{\infty}$.

A function $f \in \ell_{\infty}(G)$ is right uniformly continuous when, if for every $\epsilon>0$, there exists a neighbourhood $U$ of $e$ such that

$$
|f(s)-f(t)|<\epsilon \quad \text { whenever } \quad s t^{-1} \in U .
$$


The algebra of right uniformly continuous functions on $G$ is denoted by $\mathcal{L U C}(G)$.

A function $f \in \mathcal{C B}(G)$ is almost periodic when the set of all its left (equivalently, right) translates is a relatively norm compact subset in $\mathcal{C B}(G)$. The algebra of almost periodic functions on $G$ is denoted by $\mathcal{A P}(G)$.

A function $f \in \mathcal{C B}(G)$ is weakly almost periodic when the set of all its left (equivalently, right) translates makes a relatively weakly compact subset in $\mathcal{C B}(G)$. The algebra of weakly almost periodic functions on $G$ is denoted by $\mathcal{W} \mathcal{A P}(G)$.

The Fourier-Stieltjes algebra $B(G)$ is the linear span of the set of all continuous positive definite functions on $G$. Equivalently, $B(G)$ is the space of coefficients of unitary representations of $G$ when $G$ is locally compact. As the Fourier-Stieltjes algebra is not uniformly closed we will work with the Eberlein algebra $\mathcal{B}(G)$, which is the uniform closure of $B(G)$, in symbols $\mathcal{B}(G)=\overline{B(G)}\|\cdot\|_{\infty}$.

Let $\mu$ be the unique invariant mean on $\mathcal{W} \mathcal{A P}(G)$ (see [5], or [9]). As stated above, put

$$
\begin{aligned}
\mathcal{W A P}_{0}(G) & =\{f \in \mathcal{W} \mathcal{A P}(G): \mu(|f|)=0\}, \\
\mathcal{B}_{0}(G) & =\{f \in \mathcal{B}(G): \mu(|f|)=0\} .
\end{aligned}
$$

In [13, page 143], Chou denoted $B(G) \cap \mathcal{W} \mathcal{A} \mathcal{P}_{0}(G)$ by $B_{c}(G)$, and observed that $\mathcal{B}_{0}(G)=\overline{B_{c}(G)}$ when $G$ is locally compact.

1.2. The spectrum as a compactification. Let $G$ be a topological group, $\mathcal{A}(G) \subseteq \ell_{\infty}(G)$ be a unital $C^{*}$-subalgebra and denote by $G^{\mathcal{A}}$ the the spectrum (the set of non-zero multiplicative linear functionals) of $\mathcal{A}(G)$. Equipped with the topology of pointwise convergence, $G^{\mathcal{A}}$ becomes a compact Hausdorff topological space. There is a canonical morphism $\epsilon_{\mathcal{A}}: G \rightarrow G^{\mathcal{A}}$ given by evaluations

$$
\epsilon_{\mathcal{A}}(s)(f)=f(s) \text {, for every } f \in \mathcal{A}(G) \text { and } s \in G .
$$

This map is continuous if and only if $\mathcal{A}(G) \subseteq \mathcal{C} \mathcal{B}(G)$, and injective on $G$ if and only if $\mathcal{A}(G)$ separates the points of $G$. We may recall, for example, that the map $\epsilon_{\mathcal{A}}$ is injective on $G$ (and in fact a homeomorphism onto its image in $G^{\mathcal{A}}$ ) whenever $C_{0}(G) \subseteq \mathcal{A}(G)$. This is not a necessary condition since it may also happen that $\epsilon_{\mathcal{A}}$ is injective when $C_{0}(G) \cap \mathcal{A}(G)=\{0\}$ as it is the case when $G$ is a locally compact, maximally almost periodic and $\mathcal{A}(G)=\mathcal{A P}(G)$. It may also happen that $\epsilon_{\mathcal{A}}$ is injective on a given subset $T$ of $G$. We will then identify $T$ as a subset of $G^{\mathcal{A}}$. This situation occurs when for example $T$ is an $\mathcal{A}(G)$-interpolation set.

The $C^{*}$-algebra $\mathcal{A}(G)$ is left translation invariant when $f_{s} \in \mathcal{A}(G)$ for every $f \in \mathcal{A}(G)$ and $s \in G$. In this case for every $x \in G^{\mathcal{A}}$ and $f \in \mathcal{A}(G)$ we may define a function $x f$ on $G$ by $x f(s)=x\left(f_{s}\right)$. A translation invariant $C^{*}$-subalgebra $\mathcal{A}(G)$ of $\mathcal{C} \mathcal{B}(G)$ containing 1 , and $x f$ for every $f \in \mathcal{A}(G)$ and $x \in G^{\mathcal{A}}$ is called $m$-admissible in [5, Definition 2.10]. For simplicity we will use the term admissible algebra instead. 
When $\mathcal{A}(G)$ is an admissible $C^{*}$-subalgebra of $\mathcal{C} \mathcal{B}(G), G^{\mathcal{A}}$ can be equipped with the product $G^{\mathcal{A}}$ given by

$$
x y(f)=x(y f) \quad \text { for every } \quad x, y \in G^{\mathcal{A}} \text { and } f \in \mathcal{A}(G) .
$$

$G^{\mathcal{A}}$ then becomes a semigroup compactification of the topological group $G$ in the sense of [5]. This means that $G^{\mathcal{A}}$ is a compact semigroup having a continuous, dense, homomorphic, image of $G$ such that the mappings

$$
x \mapsto x y: G^{\mathcal{A}} \rightarrow G^{\mathcal{A}} \text { and } x \mapsto \epsilon_{\mathcal{A}}(s) x: G^{\mathcal{A}} \rightarrow G^{\mathcal{A}}
$$

are continuous for every $y \in G^{\mathcal{A}}$ and $s \in G$.

The algebras $C_{0}(G) \oplus \mathbb{C}, \mathcal{A P}(G), C_{0}(G) \oplus \mathcal{A P}(G), \mathcal{B}(G), \mathcal{W} \mathcal{A} \mathcal{P}_{0}(G) \oplus \mathbb{C}$, $\mathcal{W} \mathcal{A P}(G)$ and $\mathcal{L U C}(G)$ are all known to be admissible, see for example [5]. But when $G$ is locally compact, $\mathcal{C B}(G)$ is not admissible unless $G$ is either discrete or compact, see [3] or [28] for more.

When $G$ is a locally compact group and $\mathcal{A}$ is an admissible $C^{*}$-subalgebra of $\mathcal{L U}(G)$, the semigroup compactification $G^{\mathcal{A}}$ has the the joint continuity property, that is, the map

$$
(s, x) \mapsto \epsilon_{\mathcal{A}}(s) x: G \times G^{\mathcal{A}} \rightarrow G^{\mathcal{A}}
$$

is continuous.

A recent account on semigroup compactifications is given in [29].

1.3. A few words on notation. All our groups will be multiplicative and their identity element will be denoted as $e$. The characteristic function of a set $T$ will be denoted as $1_{T}$. If $X$ is a set and $T \subseteq X$, given $f \in \ell_{\infty}(X)$, we define $\|f\|_{T}=\sup \{|f(x)|: x \in T\}$ so that $\|f\|_{\infty}=\|f\|_{X}$. The morphism $\epsilon_{\mathcal{A}}$ maps $G$ into $G^{\mathcal{A}}$ faithfully if $\mathcal{A}(G)$ separates points. If $X \subseteq G$, we will denote the closure of $\epsilon_{\mathcal{A}}(X)$ simply as $\bar{X}^{\mathcal{A}}$, while the closure of $X$ in $G$ will be denoted as $\bar{X}$. The reason for this is that in most of our applications the algebra $\mathcal{A}(G)$ separates points of $G$ and therefore $\epsilon_{\mathcal{A}}$ may be used to identify $G$ with a subset of $G^{\mathcal{A}}$.

A standard application of Gelfand duality identifies $\mathcal{A}(G)$ with $\mathcal{C} \mathcal{B}\left(G^{\mathcal{A}}\right)$. Under this identification, to every $f \in \mathcal{A}(G)$ there corresponds $f^{\mathcal{A}} \in \mathcal{C} \mathcal{B}\left(G^{\mathcal{A}}\right)$ in such a way that the following diagram commutes

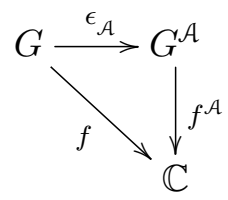

When $\epsilon_{\mathcal{A}}$ is injective $f^{\mathcal{A}}$ can be seen as an extension of $f$ to $G^{\mathcal{A}}$.

\section{INTERPOLATION SETS AND QUOTIENTS OF FUNCTION SPACES}

We begin our work by introducing in precise terms the sets we will be using, and then we prove the impact they have in measuring the size of our quotient spaces $\mathcal{A}_{2}(G) / \mathcal{A}_{1}(G)$. This is achieved in Theorem 2.11.

It is worthwhile to note that this theorem may also be applied to obtain most (if not all) of the results concerning the quotient spaces of the various function algebras mentioned in the introduction; it is of course necessary at each time to construct the required interpolation sets. 
Our final main results in this section and in the rest of the paper concern $C^{*}$-algebras of bounded functions on a locally compact group, but definitions and properties shall also be proved for a general Hausdorff topological group whenever this makes sense.

Definition 2.1. Let $G$ be a topological group and $\mathcal{A}(G) \subseteq \ell_{\infty}(G)$. A subset $T \subseteq G$ is said to be

(i) an $\mathcal{A}(G)$-interpolation set if every bounded function $f: T \rightarrow \mathbb{C}$ can be extended to a function $\bar{f}: G \rightarrow \mathbb{C}$ such that $\bar{f} \in \mathcal{A}(G)$.

(ii) an approximable $\mathcal{A}(G)$-interpolation set if it is an $\mathcal{A}(G)$-interpolation set and for every neighbourhood $U$ of $e$, there is an open neighbourhood $V$ of $e$ with $\bar{V} \subseteq \operatorname{int}(U)$ such that, for each $T_{1} \subseteq T$ there is $h \in \mathcal{A}(G)$ with $h\left(V T_{1}\right)=\{1\}$ and $h\left(G \backslash\left(U T_{1}\right)\right)=\{0\}$.

Remark 2.2. $\mathcal{A}(G)$-interpolation sets for some concrete algebras $\mathcal{A}(G) \subseteq$ $\ell_{\infty}(G)$ have been a frequent object of study, see [29] and [24] for more details and references. See also [30] for the most recent account on the subject.

Approximable interpolation sets appear in the early 70's as a crucial step in Drury's proof of the union theorem of Sidon sets, see [18]. Other wellknown interpolation sets are also approximable as for instance translationfinite sets considered by Ruppert in [50] (and called $R_{W}$-sets by Chou in [14]) that turn to be the approximable $\mathcal{W} \mathcal{A P}(G)$-interpolation sets of discrete groups, see [24] for more on this respect.

When $G$ is discrete, the definition of approximable $\mathcal{A}(G)$-interpolation set is much simpler. In that case $T \subset G$ is an approximable $\mathcal{A}(G)$-interpolation set if and only if $T$ is an $\mathcal{A}(G)$-interpolation such that $1_{T} \in \mathcal{A}(G)$, or equivalently, if every bounded function supported on $T$ is in $\mathcal{A}(G)$.

It should however be reminded that approximable $\mathcal{A}(G)$-interpolation sets do not make sense for every $C^{*}$-subalgebra $\mathcal{A}(G)$ of $\ell_{\infty}(G)$. For example, no subset in a non-compact locally compact group can be an approximable $\mathcal{A P}(G)$-interpolation set, see [24, Section 3 and Corollary 4.24].

2.1. The quotients. The following lemma contains some elementary consequences of the definitions of interpolation and approximable interpolation sets. The identification of $\bar{T}^{\mathcal{A}}$ with the Stone-Čech compactification of $T$ (with the discrete topology) allows us to use the powerful property of extreme disconnectedness of the latter compactification. As the reader will quickly notice this is the key in the arguments leading to the main results in this section. The main results start with a generalization of a theorem proved by Chou [13] for $B(G)$ (Lemma 2.5) to arbitrary $C^{*}$-subalgebras of $\ell_{\infty}(G)$. Along with some rather technical lemmas, this provides us with the conditions stated in Theorem 2.11 and Corollary 2.12 under which the quotient $\mathcal{A}_{2}(G) / \mathcal{A}_{1}(G)\left(\mathcal{A}_{1}(G) \subset \mathcal{A}_{2}(G)\right.$ being admissible $C^{*}$-subalgebras of $\mathrm{eB}(G))$ contains a linear isometric copy of $\ell_{\infty}(\kappa)$ for some cardinal $\kappa$.

Lemma 2.3. Let $G$ be a topological group. Let $\mathcal{A}(G)$ be a $C^{*}$-subalgebra of $\ell_{\infty}(G)$ with $1 \in \mathcal{A}(G)$ and $T \subseteq G$.

(i) $T$ is an $\mathcal{A}(G)$-interpolation set if and only if $\epsilon_{\mathcal{A}}$ is injective on $T$ and the canonical embedding of $T$ is a homeomorphism between $\bar{T}^{\mathcal{A}}$ 
and $\beta T_{d}$, the Stone-Čech-compactification of $T$ equipped with the discrete topology, that leaves the points of $T$ fixed.

(ii) $T$ is an $\mathcal{A}(G)$-interpolation set if and only if for every pair of subsets $T_{1}, T_{2} \subset T, T_{1} \cap T_{2}=\emptyset$ implies ${\overline{T_{1}}}^{\mathcal{A}} \cap{\overline{T_{2}}}^{\mathcal{A}}=\emptyset$.

(iii) If $T$ is an $\mathcal{A}(G)$-interpolation set and $f: T \rightarrow \mathbb{C}$ is a bounded function, then $f$ has an extension $\bar{f} \in \mathcal{A}(G)$ with $\|\bar{f}\|_{\infty}=\|f\|_{T}$.

(iv) If $T$ is an approximable $\mathcal{A}(G)$-interpolation set, then for every bounded function $h: T \rightarrow \mathbb{C}$ and every neighbourhood $U$ of the identity, there is $f \in \mathcal{A}(G)$ such that

$$
\left.f\right|_{T}=h, \quad f(G \backslash U T)=\{0\} \quad \text { and } \quad\|f\|_{\infty}=\|h\|_{T} .
$$

Proof. First observe that $\epsilon_{\mathcal{A}}$ is injective on every $\mathcal{A}(G)$-interpolation set $T$ : if $t_{1} \neq t_{2} \in T$, there is $f \in \ell_{\infty}(T)$ with $f\left(t_{1}\right) \neq f\left(t_{2}\right)$. Take $\bar{f} \in \mathcal{A}(G)$ extending $f$. By $(1) \bar{f}=\bar{f}^{\mathcal{A}} \circ \epsilon_{\mathcal{A}}$, hence $\epsilon_{A}\left(t_{1}\right) \neq \epsilon_{\mathcal{A}}\left(t_{2}\right)$.

Assertion (i) follows then from the universal property defining the StoneCech compactification of a discrete space. In fact, the restriction of the evaluation map $\epsilon_{\mathcal{A}}$ to $T$ gives a homeomorphism of the discrete set $T_{d}$ onto its image in $G^{\mathcal{A}}$. So $\bar{T}^{\mathcal{A}}$ is a (topological) compactification of $T_{d}$, and we may apply [21, Corollary 3.6.3].

Assertion (ii) follows also directly from a well-known characterization of the Stone-Cech compactification of a discrete space, see for instance [21, Corollary 3.6.2].

To prove (iii), let $f: T \rightarrow \mathbb{C}$ with $\|f\|_{T}=M$ be given. If $B_{M}$ is the closed disc of radius $M$ centered at 0 (in $\mathbb{C}$ ), we can use (i) and the universal property of $\beta T_{d}$ to find a continuous function $f^{\beta}: \bar{T}^{\mathcal{A}} \rightarrow B_{M}$ with $\left.f^{\beta}\right|_{T}=f$. Then, by Tietze's extension theorem, $f^{\beta}$ can be extended to a continuous function $f^{\mathcal{A}}: G^{\mathcal{A}} \rightarrow B_{M}$, the restriction $\left.f^{\mathcal{A}}\right|_{G}$ is then the desired extension.

To prove (iv), let $T$ be an approximable $\mathcal{A}(G)$-interpolation set. First, we find, using (iii), $f_{1} \in \mathcal{A}$ with $\left.f_{1}\right|_{T}=h$ and $\left\|f_{1}\right\|_{\infty}=\|h\|_{T}$. The definition of approximable $\mathcal{A}(G)$-interpolation sets provides a neighbourhood $V$ with $\bar{V} \subseteq \operatorname{int}(U)$ and $f_{2} \in \mathcal{A}$ such that

$$
f_{2}(V T)=\{1\} \quad \text { and } \quad f_{2}(G \backslash U T)=\{0\} .
$$

Using [21, 3.2.20], we can assume (taking the minimum of $f_{2}$ and the function that is constant and equal to 1 ) that $\left\|f_{2}\right\|_{\infty}=1$. The product $f_{1} \cdot f_{2}$ then coincides with $h$ on $T$ and vanishes off $U T$.

Remark 2.4. Note that if in the lemma above $\mathcal{A}(G) \subseteq \mathcal{C B}(G)$, then $T$ is necessarily discrete since every bounded function on $T$ must be continuous.

Observe as well that the sole existence of an infinite $\mathcal{A}(G)$-interpolation set $T$ in $G$, implies that $G^{\mathcal{A}}$ contains a copy of $\beta T_{d}$, where $T_{d}$ is the discrete set $T$. The compactification $G^{\mathcal{A}}$ is therefore large and topologically involved.

The following theorem, due to Chou [13], has its roots in a result of Ramirez (see Theorem 2.3 of [19]) in the Abelian setting. This theorem is used by Chou, loc. cit., to find an isometric copy of $\ell_{\infty}$ inside $\mathcal{W} \mathcal{A P}(G) / \mathcal{B}(G)$ for a discrete group $G$. This was originally the departing point of our paper.

Theorem 2.5. (Chou, [13, Lemma 3.11]) Let $G$ be a discrete group. A subset $T \subseteq G$ fails to be a $B(G)$-interpolation set if and only if there is a 
bounded function $f \in \ell_{\infty}(G)$, with $\|f\|_{\infty}=1$ such that

$$
\|\phi-f\|_{T} \geq 1 \quad \text { for all } \phi \in B(G) .
$$

Remark 2.6. It is an immediate consequence of the previous theorem that $\mathcal{B}(G)$-interpolation sets are also $B(G)$-interpolation sets (i.e., Sidon sets). We do not know whether Theorem 2.5 remains valid for all locally compact groups.

The result in Theorem 2.5 is more natural when the function algebra is a $C^{*}$-subalgebra. It is not surprising therefore that it holds for any $C^{*}$ subalgebra. Next lemma proves even more.

Lemma 2.7. Let $G$ be a topological group, $\mathcal{A}_{1}(G) \subseteq \mathcal{A}_{2}(G) \subseteq \ell_{\infty}(G)$ be two $C^{*}$-subalgebras with $1 \in \mathcal{A}_{1}(G)$, and let $\left(T_{\eta}\right)_{\eta<\kappa}$ be a family of disjoint subsets of $G$ such that

(i) each $T_{\eta}$ fails to be an $\mathcal{A}_{1}(G)$-interpolation set,

(ii) $T=\bigcup_{\eta<\kappa} T_{\eta}$ is an approximable $\mathcal{A}_{2}(G)$-interpolation set.

Then for each open neighbourhood $U$ of e, there is a function $f \in \mathcal{A}_{2}(G)$ with $\|f\|_{\infty}=1$ such that

$f(G \backslash U T)=\{0\} \quad$ and $\quad\|f-\phi\|_{T_{\eta}} \geq 1$ for every $\eta<\kappa$ and every $\phi \in \mathcal{A}_{1}(G)$.

Proof. Let $T=\bigcup_{\eta<\kappa} T_{\eta}$ be an approximable $\mathcal{A}_{2}(G)$-interpolation set as stated in the lemma. Let $U$ be an open neighbourhood of $e$.

To avoid cumbersomeness, we abuse our notation and use the same letters to denote subsets of $T$ and their images in $G^{\mathcal{A}_{1}}$.

Then, by Statement (ii) of Lemma 2.3, each $T_{\eta}$ must contain two disjoint subsets $T_{1, \eta}, T_{2, \eta}$ such that $\overline{T_{1, \eta}} \mathcal{A}_{1} \cap \overline{T_{2, \eta}} \mathcal{A}_{1} \neq \emptyset$. Define for each $\eta<\kappa$, a function $h_{\eta}: G \rightarrow[-1,1]$ with $h_{\eta}\left(T \backslash T_{\eta}\right)=\{0\}$,

$$
h_{\eta}\left(T_{1, \eta}\right)=\{1\} \quad \text { and } \quad h_{\eta}\left(T_{2, \eta}\right)=\{-1\} .
$$

Then consider the function $h: G \rightarrow[-1,1]$ given by

$$
h(t)=h_{\eta}(t) \text { if } t \in T_{\eta} \text { for some } \eta<\kappa \text { and } h(G \backslash T)=\{0\} .
$$

By Statement (iv) of Lemma 2.3, there is a a function $f \in \mathcal{A}_{2}(G)$ such that

$$
f(G \backslash U T)=0,\left.\quad f\right|_{T}=h \text { and }\|f\|_{\infty}=\|h\|_{T}=1 .
$$

Let now $\phi$ be any function in $\mathcal{A}_{1}(G)$, and take $\varepsilon>0$. Given $\eta<\kappa$, we are going to prove that $\|f-\phi\|_{T_{\eta}} \geq 1-\varepsilon$.

Take $p_{\eta} \in{\overline{T_{1, \eta}}}^{\mathcal{A}_{1}} \cap \overline{T_{2, \eta}} \mathcal{A}_{1}$ and pick $t_{1, \eta} \in T_{1, \eta}$ and $t_{2, \eta} \in T_{2, \eta}$ with

$$
\left|\phi\left(t_{1, \eta}\right)-\phi^{\mathcal{A}_{1}}\left(p_{\eta}\right)\right|<\varepsilon \quad \text { and }\left|\phi\left(t_{2, \eta}\right)-\phi^{\mathcal{A}_{1}}\left(p_{\eta}\right)\right|<\varepsilon \text {, }
$$

where $\phi^{\mathcal{A}_{1}}$ denotes the extension of $\phi$ to $G^{\mathcal{A}_{1}}$. Then

$$
\begin{aligned}
2 & =\left|h_{\eta}\left(t_{1, \eta}\right)-h_{\eta}\left(t_{2, \eta}\right)\right|=\left|h\left(t_{1, \eta}\right)-h\left(t_{2, \eta}\right)\right|=\left|f\left(t_{1, \eta}\right)-f\left(t_{2, \eta}\right)\right| \\
& \leq\left|f\left(t_{1, \eta}\right)-\phi\left(t_{1, \eta}\right)\right| \\
& +\left|\phi\left(t_{1, \eta}\right)-\phi^{\mathcal{A}_{1}}\left(p_{\eta}\right)\right|+\left|\phi^{\mathcal{A}_{1}}\left(p_{\eta}\right)-\phi\left(t_{2, \eta}\right)\right|+\left|\phi\left(t_{2, \eta}\right)-f\left(t_{2, \eta}\right)\right| .
\end{aligned}
$$

It follows that either $\left|f\left(t_{1, \eta}\right)-\phi\left(t_{1, \eta}\right)\right| \geq 1-\varepsilon$ or $\left|f\left(t_{2, \eta}\right)-\phi\left(t_{2, \eta}\right)\right| \geq 1-\varepsilon$. Since $\varepsilon>0$ was arbitrary, we find that $\|f-\phi\|_{T_{\eta}} \geq 1$. Since $\|f\|_{\infty}=1$ and $f(G \backslash U T)=\{0\}$, we see that $f$ is the required function. 
For the main theorem in this section, we need to recall the following definitions. These sets are also essential for the rest of the paper.

Definition 2.8. Let $G$ be a topological group, $T$ be a subset of $G$ and $U$ be a neighbourhood of $e$. We say that $T$ is right $U$-uniformly discrete if

$$
U s \cap U s^{\prime}=\emptyset \quad \text { for every } \quad s \neq s^{\prime} \in T .
$$

The set $T$ being left $U$-uniformly discrete is defined analogously. We say that $T$ is right uniformly discrete (resp. left uniformly discrete) when it is right $U$ uniformly discrete (resp. left $U$-uniformly discrete) for some neighbourhood $U$ of $e$. If $T$ is both left and right uniformly discrete, we say that $T$ is uniformly discrete.

Lemma 2.9. Let $G$ be a locally compact group, $\mathcal{A}(G)$ be a $C^{*}$-subalgebra of $\mathcal{C B}(G), U$ be a compact neighbourhood of e, and $T \subseteq G$ be an approximable $\mathcal{A}(G)$-interpolation set that can be partitioned as $T=\bigcup_{\eta<\kappa} T_{\eta}$ with $U T_{\eta} \cap$ $U T_{\eta^{\prime}}=\emptyset$ whenever $\eta \neq \eta^{\prime}$. Then there is a compact neighbourhood $V$ of the identity with $V^{2} \subseteq U$ such that given any two functions $f, g \in \ell_{\infty}(G)$ that are 0 off $V T$ and a function $\mathbf{c}=\left(c_{\eta}\right)_{\eta<\kappa} \in \ell_{\infty}(\kappa)$ such that

$$
\left.f\right|_{V T_{\eta}}=\left.c(\eta) g\right|_{V T_{\eta}} \text { for each } \eta<\kappa,
$$

one has that:

(i) If $g$ is continuous, then so is $f$.

(ii) If $T$ is right $U$-uniformly discrete, $\mathcal{A}(G)$ is an admissible $C^{*}$-subalgebra of $\mathcal{L U C}(G)$, then $g \in \mathcal{A}(G)$, implies $f \in \mathcal{A}(G)$.

Proof. First, consider a neighbourhood $U_{0}$ of $e$ with $U_{0}^{2} \subseteq U$ and the neighbourhood $V$ provided by the definition of approximable $\mathcal{A}(G)$-interpolation sets for $U_{0}$. Then $V^{2} \subseteq U$.

Let $g \in \mathcal{C B}(G)$ and $f \in \ell_{\infty}(G)$ be functions with $f(G \backslash V T)=g(G \backslash V T)=$ $\{0\}$, related as in (3). We prove that $f$ is continuous considering separately continuity at interior points of $V T$ and points that do not belong to the interior of $V T$.

Let $s \in G$ that is not an interior point of $V T$. Since $s$ can be approached from $G \backslash V T$, we see by continuity that $g(s)=0$. By checking the cases when $s \in V T$ and when $s \notin V T$, we deduce that $f(s)=0$ as well. Now let $\left(x_{\alpha}\right)$ be a net in $G$ converging to $s$. We can assume that either $\left(x_{\alpha}\right) \subset V T$ or $\left(x_{\alpha}\right) \subset G \backslash V T$. In the former case, we use the fact that $\left|f\left(x_{\alpha}\right)\right| \leq$ $\|\mathbf{c}\|_{\infty} \cdot\left|g\left(x_{\alpha}\right)\right|$ and conclude that $\lim _{\alpha} f\left(x_{\alpha}\right)=0=f(s)$. In the other case when $\left(\left(x_{\alpha}\right) \subset G \backslash V T\right)$, it is clear that $\lim _{\alpha} f\left(x_{\alpha}\right)=0=f(s)$. The continuity of $f$ at $s$ follows.

Suppose now that $s$ is an interior point of $V T$. Pick $\eta<\kappa$ such that $s=v t$ with $v \in V$ and $t \in T_{\eta}$, and let $W$ be a symmetric neighbourhood of the identity with $W s \subseteq V T$ such that $\left|g(s)-g\left(s^{\prime}\right)\right|<\epsilon$ for every $s^{\prime} \in W s$.

Let $x \in W s$. There is then $w \in W$ with $x=w s=w v t$. Whenever $x=v_{0} t_{0}$ for some $v_{0} \in V$ and $t_{0} \in T$, one always has that $t_{0} \in T_{\eta}$ (because $V^{2} \subset U$ and $\left.U T_{\eta} \cap U T_{\eta^{\prime}}=\emptyset\right)$. Therefore,

$$
|f(s)-f(x)|=|c(\eta) g(s)-c(\eta) g(x)| \quad \text { for every } \quad x \in W s,
$$

and the continuity of $f$ at interior points follows as well. 
We now assume $\mathcal{A} \subseteq \mathcal{L U C}(G)$. Define a function $\varphi$ on $T$ by $\varphi(t)=c(\eta)$ for every $t \in T_{\eta}$. Since $T$ is an $\mathcal{A}(G)$-interpolation set, we may extend $\varphi$ to a function $\bar{\varphi} \in \mathcal{A}(G)$. By Lemma 2.3 (iv), we can assume that $\bar{\varphi}(G \backslash V T)=$ $\{0\}$. If $g^{\mathcal{A}}$ and $\varphi^{\mathcal{A}}$ denote the respective extensions of $g$ and $\bar{\varphi}$ to $G^{\mathcal{A}}$, we define $f^{*}: G^{\mathcal{A}} \rightarrow \mathbb{C}$ by

$$
\begin{aligned}
f^{*}(v p) & =\varphi^{\mathcal{A}}(p) \cdot g^{\mathcal{A}}(v p) \text { if } v \in V \text { and } p \in \bar{T}^{\mathcal{A}} \\
f^{*}(x) & =0 \text { if } x \notin V \bar{T}^{\mathcal{A}} .
\end{aligned}
$$

We check that $f^{*}$ is a well-defined, continuous extension of $f$ to $G^{\mathcal{A}}$.

(1) $f^{*}$ is well defined. It might happen that some $v p \in V \bar{T}^{\mathcal{A}}$ admits two different decompositions. We check that the definition of $f^{*}$ does not depend of the choice of the decomposition. Suppose therefore that $v_{1} p_{1}=v_{2} p_{2}$ with $v_{1}, v_{2} \in V$ and $p_{1}, p_{2} \in \bar{T}^{\mathcal{A}}$.

If $p_{1} \neq p_{2}$, we may choose $T_{1}, T_{2} \subset T$ such that

$$
{\overline{T_{1}}}^{\mathcal{A}} \cap{\overline{T_{2}}}^{\mathcal{A}}=\emptyset, p_{1} \in{\overline{T_{1}}}^{\mathcal{A}} \quad \text { and } \quad p_{2} \in{\overline{T_{2}}}^{\mathcal{A}}
$$

(this is possible by (ii) of Lemma 2.3). Since $T$ is approximable, we may pick $h \in \mathcal{A}(G)$ such that

$$
h\left(V T_{1}\right)=\{1\} \quad \text { and } \quad h\left(G \backslash \operatorname{int}\left(U_{2}\right) T_{1}\right)=\{0\} .
$$

Recalling that multiplication by elements of $G$ is continuous on $G^{\mathcal{A}}$, it is clear that $h^{\mathcal{A}}\left(v_{1} p_{1}\right)=1$. By the same reason, if $\left(t_{\alpha}\right)$ is a net in $T_{2}$ converging to $p_{2}$, we have that $v_{2} p_{2}=\lim _{\alpha} v_{2} t_{\alpha}$. But since $T$ is right $U$ uniformly discrete, no element $v_{2} t_{\alpha}$ can be in $V_{2} T_{1}$, hence $h^{\mathcal{A}}\left(v_{2} p_{2}\right)$ must be zero. This contradiction shows that

$$
v_{1} p_{1}=v_{2} p_{2} \text { implies } p_{2}=p_{1} \text {. }
$$

This shows already that $f^{*}$ is well defined, since the equalities $v_{1} p_{1}=v_{2} p_{2}$ and $p_{1}=p_{2}$ give us

$$
f^{*}\left(v_{1} p_{1}\right)=\varphi^{\mathcal{A}}\left(p_{1}\right) g^{\mathcal{A}}\left(v_{1} p_{1}\right)=f^{*}\left(v_{2} p_{2}\right) .
$$

(In fact, $v_{1}$ and $v$ must be also equal by the same argument, but this is enough for our purposes.)

(2) $f^{*}$ coincides with $f$ on $G$. Since $V \bar{T}^{\mathcal{A}} \cap G=V\left(\bar{T}^{\mathcal{A}} \cap G\right)=V T$ and $f(G \backslash V T)=\{0\}$, we readily see that $f$ and $f^{*}$ coincide on $G \backslash V T$. Let on the other hand $s=v t$ with $v \in V$ and $t \in T_{\eta}$. Then

$$
f^{*}(s)=\varphi(t) g(s)=c(\eta) g(s)=f(s) .
$$

(3) $f^{*}$ is continuous. Using the joint continuity property, we see that $V \bar{T}^{\mathcal{A}}$ is closed in $G^{\mathcal{A}}$. So the continuity of $f^{*}$ at the points outside of $V \bar{T}^{\mathcal{A}}$ is clear.

We divide the case $x=v p \in V \bar{T}^{\mathcal{A}}$ into two subcases. Suppose first that $x$ is an interior point $V \bar{T}^{\mathcal{A}}$, and let $\left(q_{\alpha}\right)$ be a net in $G^{\mathcal{L} u \mathcal{C}}$ converging to $x$. Then $\left(q_{\alpha}\right)$ is eventually of the form $\left(v_{\alpha} p_{\alpha}\right)$ with $\left(v_{\alpha}\right)$ in $V$ and $\left(p_{\alpha}\right)$ in $\bar{T}^{\mathcal{A}}$. By taking subnets if necessary, we may assume that $\lim _{\alpha} v_{\alpha}=v_{0}$ in $V$ and 
$\lim _{\alpha} p_{\alpha}=p_{0}$ in $\bar{T}^{\mathcal{A}}$. Accordingly, $x=v p=v_{0} p_{0}$, and applyig 5 , we see that $p=p_{0}$. Therefore,

$$
f^{*}\left(q_{\alpha}\right)=\varphi^{\mathcal{A}}\left(p_{\alpha}\right) g^{\mathcal{A}}\left(q_{\alpha}\right) \longrightarrow \varphi^{\mathcal{A}}\left(p_{0}\right) g^{\mathcal{A}}(x)=\varphi^{\mathcal{A}}(p) g^{\mathcal{A}}(x)=f^{*}(x),
$$

as required. The second subcase is when $x$ is outside the interior of $V \bar{T}^{\mathcal{A}}$. Here, we may assume that the net $\left(q_{\alpha}\right)$ given to converge to $x$ is also outside $V \bar{T}^{\mathcal{A}}$, and so $g^{\mathcal{A}}\left(q_{\alpha}\right)=0$ for every $\alpha$. Since $g^{\mathcal{A}}$ is continuous, we deduce that $f^{*}(x)=\varphi^{\mathcal{A}}(p) g^{\mathcal{A}}(x)=0$, as required.

From (1), (2), (3) we conclude that $f \in \mathcal{A}$.

Remarks 2.10. (i) A known theorem due to Veech asserts that the left action of a locally compact group $G$ on $G^{\mathcal{L} U \mathcal{C}}$ is free, i.e., $g x \neq x$ for every $x \in G^{\mathcal{L U C}}$ and $g \in G, g \neq e$, see [52], or [46] for a shorter proof. The proof of the previous Lemma reveals that Veech's property in fact holds in $G^{\mathcal{A}}$ at any point in the closure of the approximable $\mathcal{A}(G)$-interpolation sets with $\mathcal{A} \subset \mathcal{L U} \mathcal{U}(G)$. That is, if $T$ is any such a set, $x \in \bar{T}^{\mathcal{A}}$ and $g \neq e$ in $G$, then $g x \neq x$ and $x g \neq x$ in $G^{\mathcal{A}}$. This property was proved in $G^{\mathcal{W A P}}$ in [4] and [23] using $t$-sets. $t$-Sets are by [24] approximable $\mathcal{W} \mathcal{A P}(G)$-interpolation sets. We will return to these matters in a forthcoming work.

(ii) It could also be worth to mention that for metrizable locally compact groups the condition on $T$ in (ii) is redundant. Indeed, by [24, Theorem 4.9], every $\mathcal{L U C}(G)$-interpolation subset of a metrizable group is right uniformly discrete.

Theorem 2.11. Let $G$ be a locally compact group and let $\mathcal{A}_{1}(G) \subset \mathcal{A}_{2}(G) \subseteq$ $\mathcal{L U C}(G)$ be two unital $C^{*}$-subalgebras of $\ell_{\infty}(G)$ with $\mathcal{A}_{2}(G)$ admissible. Let, in addition, $U$ be a compact neighbourhood of the identity such that $T$ is right $U$-uniformly discrete. Suppose that $G$ contains a family of sets $\left\{T_{\eta}: \eta<\kappa\right\}$ such that

(i) $T_{\eta} \cap T_{\eta^{\prime}}=\emptyset$ for every $\eta \neq \eta^{\prime}<\kappa$,

(ii) $T_{\eta}$ fails to be an $\mathcal{A}_{1}(G)$-interpolation set for every $\eta<\kappa$, and

(iii) $T=\bigcup_{\eta<\kappa} T_{\eta}$ is an approximable $\mathcal{A}_{2}(G)$-interpolation set.

Then there is a linear isometry $\Psi: \ell_{\infty}(\kappa) \rightarrow \mathcal{A}_{2}(G) / \mathcal{A}_{1}(G)$.

Proof. Let $V$ be the neighbourhood of the identity provided by Lemma 2.9.

Since $T=\bigcup_{\eta<\kappa} T_{\eta}$ is an approximable $\mathcal{A}_{2}(G)$-interpolation set and each $T_{\eta}$ fails to be an $\mathcal{A}_{1}(G)$-interpolation set, we take from Lemma 2.7 a function $f \in \mathcal{A}_{2}(G)$ with $\|f\|_{\infty}=1$ such that

$f(G \backslash V T)=\{0\} \quad$ and $\quad\|f-\phi\|_{T_{\eta}} \geq 1 \quad$ for all $\quad \phi \in \mathcal{A}_{1}(G)$ and $\eta<\kappa$.

For each $\mathbf{c}=\left(c_{\eta}\right)_{\eta<\kappa} \in \ell_{\infty}(\kappa)$, we define the function $f_{\mathbf{c}}: G \rightarrow \mathbb{C}$ supported in $V T$ by

$$
f_{\mathbf{c}}(v t)=c_{\eta} f(v t) \quad \text { if } \quad t \in T_{\eta} \quad \text { and } \quad \eta<\kappa,
$$

i.e., with the notation of Lemma $2.9,\left.f_{\mathbf{c}}\right|_{V T_{\eta}}=\left.\mathbf{c}(\eta) f\right|_{V T_{\eta}}$.

Then $f_{\mathbf{c}} \in \mathcal{A}_{2}(G)$ by (ii) of Lemma 2.9. Obviously, the map $\Psi: \ell_{\infty}(\kappa) \rightarrow$ $\mathcal{A}_{2}(G) / \mathcal{A}_{1}(G)$ given by

$$
\Psi(\mathbf{c})=f_{\mathbf{c}}+\mathcal{A}_{1}(G) \quad \text { for every } \quad \mathbf{c} \in \ell_{\infty}(\kappa)
$$


is linear. We next check that it is isometric.

The same argument of [13, Theorem 3.12] shows now that, for every $\eta_{0}<\kappa$,

$$
\begin{aligned}
\left\|\Psi\left(\left(c_{\eta}\right)_{\eta<\kappa}\right)\right\|_{\mathcal{A}_{2}(G) / \mathcal{A}_{1}(G)} & =\inf \left\{\left\|f_{\mathbf{c}}-\phi\right\|_{\infty}: \phi \in \mathcal{A}_{1}(G)\right\} \\
& \geq \inf \left\{\left\|f_{\mathbf{c}}-\phi\right\|_{T_{\eta_{0}}}: \phi \in \mathcal{A}_{1}(G)\right\} \\
& =\inf \left\{\left\|c_{\eta_{0}} f-\phi\right\|_{T_{\eta_{0}}}: \phi \in \mathcal{A}_{1}(G)\right\} \\
& =\left|c_{\eta_{0}}\right| \inf \left\{\|f-\phi\|_{T_{\eta_{0}}}: \phi \in \mathcal{A}_{1}(G)\right\} \\
& \geq\left|c_{\eta_{0}}\right|,
\end{aligned}
$$

where the last inequality follows from the choice of $f$. Since, obviously,

$$
\|\Psi(\mathbf{c})\|_{\mathcal{A}_{2}(G) / \mathcal{A}_{1}(G)} \leq\left\|f_{\mathbf{c}}\right\|_{\infty}=\|\mathbf{c}\|, \quad \text { for every } \quad \mathbf{c}=\left(c_{\eta}\right)_{\eta<\kappa} \in \ell_{\infty}(\kappa),
$$

we see that $\Psi$ is the required isometry.

Corollary 2.12. If in the above theorem $\mathcal{A}_{2}(G)=\mathcal{C} \mathcal{B}(G)$ and $T$ is not assumed to be right $U$-uniformly discrete but still $U T_{\eta} \cap U T_{\eta^{\prime}}=\emptyset$, then the quotient $\mathrm{C} \mathcal{B}(G) / \mathcal{A}_{1}(G)$ contains a linearly isometric copy of $\ell_{\infty}(\kappa)$.

Proof. The proof of Theorem 2.11 remains valid in this case applying (i) of Lemma 2.9 instead of (ii).

Remark 2.13. Two $C^{*}$-subalgebras of $\ell_{\infty}(G)$ may be different, and yet produce a small quotient (i.e., separable), for example if $G$ is a minimally weakly almost periodic group ([14], [49], [53]) then $\mathcal{W} \mathcal{A P}(G) / \mathcal{A P}(G)=C_{0}(G)$. If $G=S L(2, \mathbb{R})$, then $\mathcal{W} \mathcal{A P}(G)=C_{0}(G) \oplus \mathbb{C} 1$, and so $\mathcal{W} \mathcal{A P}(G) / C_{0}(G)=\mathbb{C}$. In the theorem and corollary above, we have just met conditions under which this is not so.

Corollary 2.14. Under the hypotheses of Theorem 2.11 or Corollary 2.12, the quotient space $\mathcal{A}_{2}(G) / \mathcal{A}_{1}(G)$ is non-separable.

\section{INTERPOLATION SETS}

The definitions in this section gather the topological group-theoretic properties that will correspond to the interpolation sets needed in the three sections that follow. Once these interpolation sets are at hand, an application of Theorem 2.11 and Corollary 2.12 will lead immediately to the desired conclusion on the quotients.

In addition to the uniformly discrete sets defined in the previous sections we shall also need the following sets.

Definition 3.1. Let $G$ be a non-compact topological group. We say that a subset $T$ of $G$ is

(i) right translation-finite if every infinite subset $L \subseteq G$ contains a finite subset $F$ such that $\bigcap\left\{b^{-1} T: b \in F\right\}$ is finite; left translationfinite if every infinite subset $L \subseteq G$ contains a finite subset $F$ such that $\bigcap\left\{T b^{-1}: b \in F\right\}$ is finite; and translation-finite when it is both right and left translation-finite.

(ii) right translation-compact if every non-relatively compact subset $L \subseteq$ $G$ contains a finite subset $F$ such that $\bigcap\left\{b^{-1} T: b \in F\right\}$ is relatively compact; left translation-compact if every non-relatively compact 
subset $L \subseteq G$ contains a finite subset $F$ such that $\bigcap\left\{T b^{-1}: b \in F\right\}$ is relatively compact; and translation-compact when it is both left and right translation-compact.

(iii) a right t-set (left $t$-set) if there exists a compact subset $K$ of $G$ containing $e$ such that $g T \cap T$ (respectively, $T g \cap T$ ) is relatively compact for every $g \notin K$; and a $t$-set when it is both a right and a left $t$-set.

We also need to establish the range of locally compact groups to which our methods apply in the next two sections, these are those locally compact groups for which the existence of a good supply of $\mathcal{W} \mathcal{A P}$-functions is guaranteed.

Recall that a locally compact group $G$ is an $I N$-group if it has a compact invariant neighbourhood of the identity. We recall also from [10], that a locally compact group $G$ is an $E$-group if it contains a non-relatively compact set $X$ such that for each neighbourhood $U$ of $e$, the set

$$
\bigcap\left\{x^{-1} U x: x \in X \cup X^{-1}\right\}
$$

is again a neighbourhood of $e$. The set $X$ is called an E-set. This is a large class of locally compact groups. This includes of course all non-compact $S I N$-groups, the groups with a non-compact centre such as the matrix group $G L(n, \mathbb{R})$, and the direct product of any E-group with any locally compact group.

A detailed study of approximable $\mathcal{L} U \mathcal{C}$ - and $\mathcal{W} \mathcal{A P}(G)$-interpolation sets, with some precise characterizations, is carried out in the recent paper [24]. We summarize in Lemma 3.2 the results that will be needed in the present paper.

Lemma 3.2. ([24, Lemma 4.8 and Proposition 3.3 (iii)]) Let $G$ be a topological group and let $T \subset G$.

(i) If the underlying topological space of $G$ is normal, then all discrete closed subsets of $G$ are approximable $\mathcal{C B}(G)$-interpolation sets.

(ii) If $T$ is right (resp. left) uniformly discrete, then $T$ is an approximable $\mathcal{L U C}(G)$-interpolation set (resp. $\mathcal{R U}(G)$-interpolation set).

(iii) If $G$ is assumed to be metrizable, then every $\mathcal{L U C}(G)$-interpolation set (resp. $\mathcal{R U C}(G)$-interpolation set) is right (left) uniformly discrete.

(iv) If $G$ is an E-group and $T$ is an E-set in $G$ which is right (or left) uniformly discrete with respect to $U^{2}$ for some neighbourhood $U$ of the identity such that UT is translation-compact, then $T$ is an approximable $\mathcal{W A P}_{0}(G)$-interpolation set.

(v) If $G$ is a metrizable E-group, an E-set $T \subset G$ is a $\mathcal{W} \mathcal{A P}(G)$ interpolation set if and only if $U T$ is translation-compact for some compact neighbourhood $U$ of the identity such that $T$ is right (or left) uniformly discrete with respect to $U^{2}$.

The following Lemma will be needed later on in Section 5 .

Lemma 3.3. Let $G$ be a locally compact group, let $H$ be a closed subgroup of $G$ and let $T \subset H$. 
(i) If $U T$ is a right $t$-set in $H$ for some compact neighbourhood $U$ of the identity $e$ in $H$, then $V T$ is a right $t$-set in $G$ for every compact neighbourhood $V$ of $e$ in $G$.

(ii) If in addition $T$ is central, then the left analogue of Statement (i) holds also.

Proof. Let $U$ be a compact neighbourhood of $e$ in $H$, and suppose that $U T$ is a right t-set in $H$. By definition there is a compact subset $K \subseteq H$ such that $g U T \cap U T$ is relatively compact whenever $g \notin K$.

Let $V$ be a compact neighbourhood of $e$ and let $K^{\prime}=V K V^{-1}$.

Take $g \in G$ but $g \notin K^{\prime}$. Suppose $g V T \cap V T$ is not relatively compact. There is then a net $\left(g_{\alpha}\right)$ in $g V T \cap V T$ with no convergent subnet. For each $\alpha$, there are $v_{\alpha}, w_{\alpha} \in V$ and $t_{\alpha}, s_{\alpha} \in T$ such that $g_{\alpha}=v_{\alpha} t_{\alpha}=g w_{\alpha} s_{\alpha}$, and so

$$
t_{\alpha} s_{\alpha}^{-1}=v_{\alpha}^{-1} g w_{\alpha} \quad \text { for every } \quad \alpha .
$$

We can assume, by considering subnets if necessary, that $\left(v_{\alpha}\right)$ and $\left(w_{\alpha}\right)$ converge to $v, w \in V$, respectively. Therefore $\left(t_{\alpha} s_{\alpha}^{-1}\right)$ is a net in $H$ which converges to $h=v^{-1} \mathrm{gw}$. Since $H$ is closed, $h \in H$. Thus, the net $\left(t_{\alpha} s_{\alpha}^{-1}\right)$ is eventually in $h U$. This means that the net $\left(t_{\alpha}\right)$ belongs to $h U T \cap U T$. Since $\left(g_{\alpha}\right)$ has no convergent subnet and $\left(v_{\alpha}\right)$ does have it, we know that $\left(t_{\alpha}\right)$ cannot have convergent subnets either. We deduce therefore that $h U T \cap U T$ is not relatively compact. That however would imply that $h \in K$, and so that $g \in K^{\prime}$, a contradiction. Hence, $V T$ is a right $t$-set in $G$.

To prove the analogous statement for left $t$-sets, we suppose in addition that $T$ is central and put again $K^{\prime}=V K V$. Using the fact that $T$ is central, the same argument leads to

$$
s_{\alpha}^{-1} t_{\alpha}=w_{\alpha} g v_{\alpha}^{-1} \quad \text { for every } \quad \alpha .
$$

By taking subnets, we see that $\left(t_{\alpha}\right)$ is eventually in $T U h \cap T U=U T h \cap U T$ with $h \in V g V$. Thus, $h$ must be in $K$, and so $g$ is in $K^{\prime}$.

Remark 3.4. Notice that the above argument is also meaningful when $G=H$. In that case it shows the following: if $T \subset G$ is such that $U T$ is a right $t$-set for some compact neighbourhood $U$ of $e$ in $G$, then $V T$ is a right $t$-set for every compact neighbourhood $V$ of $e$ in $G$. We thank the referee for pointing this to us.

To state a well-known necessary condition for a subset $T \subseteq G$ to be a $\mathcal{B}(G)$-interpolation set we need the concept of large squares that we recall from [13, Definition 3.3]:

A finite subset $F$ of $G$ is an $n$-square if $F=A B$ where $|A|=|B|=n$ and $|F|=n^{2}$. A subset of a group is then said to contain large squares if it contains an $n$-square for every $n \in \mathbb{N}$.

Large squares are incompatible with Sidon sets, as proved in [13, Proposition 3.4]. We restate here this theorem, stressing on $\mathcal{B}(G)$.

Theorem 3.5. (Chou, [13]) Let $G$ be a topological group. A $\mathcal{B}(G)$-interpolation set cannot contain large squares.

Proof. Let $G_{d}$ be the group $G$ with the discrete topology. If $T$ is a $\mathcal{B}(G)$ interpolation set, then $T$ is also a $\mathcal{B}\left(G_{d}\right)$-interpolation set. By Remark 2.6, 
$T$ is then a $B\left(G_{d}\right)$-interpolation set and, by [13, Proposition 3.4], $T$ cannot contain large squares.

\section{The QUOTIEnts of $\mathcal{W} \mathcal{A} \mathcal{P}_{0}(G)$ BY $C_{0}(G)$ AND $\mathcal{W} \mathcal{A P}(G)$ ву $\mathcal{A P}(G) \oplus C_{0}(G)$}

In [10], Chou considered $E$-groups and proved that the quotient space $\mathcal{W} \mathcal{A} \mathcal{P}_{0}(G) / C_{0}(G)$ contains a linear isometric copy of $\ell_{\infty}$. In this section, we strengthen this result and prove that if $G$ is an $E$-group, then there is a linear isometric copy of $\ell_{\infty}(\kappa)$ in the quotient $\mathcal{W A P}_{0}(G) / C_{0}(G)$ where $\kappa$ is the compact covering number of an $E$-set contained in $G$. In particular, $\kappa=\kappa(G)$ when $G$ is an $S I N$-group.

Our method applies further to show that the quotient $\mathcal{W} \mathcal{A P}(G) / \mathcal{A P}(G) \oplus$ $C_{0}(G)$ is non-separable.

Theorem 4.1. Let $G$ be a non-compact locally compact E-group having an $E$-set $X$ with a compact covering number $\kappa$. Then the quotient space $\mathcal{W A P}_{0}(G) / C_{0}(G)$ contains a linear isometric copy of $\ell_{\infty}(\kappa)$.

Proof. Let $V$ be a fixed compact symmetric neighborhood of $e$. Then we consider a set $T \subset X$ as that constructed in Section 2 of [23]. This set has the following properties:

(i) $\kappa(T)=|T|=\kappa(X)$.

(ii) $T$ is right $V^{2}$-uniformly discrete.

(iii) $V T$ is a $t$-set.

For completeness, we recall from [23] the construction of the set $T$ since this shall be needed in the proof. We may assume that $e \in X$ and start with $x_{0}=e$. Suppose that the elements $x_{\beta}$ have been selected for all $\beta<\alpha$ with $\alpha<\kappa$. Set

$$
X_{\alpha}=\bigcup_{\beta_{1}, \beta_{2}, \beta_{3}<\alpha} V^{2} x_{\beta_{1}}^{\epsilon_{1}} x_{\beta_{2}}^{\epsilon_{2}} V^{2} x_{\beta_{3}}^{\epsilon_{3}} V,
$$

where each $\epsilon_{i}= \pm 1$. Since $\kappa\left(X_{\alpha}\right)<\kappa$, we pick $x_{\alpha}$ in $X \backslash X_{\alpha}$ for our set $T$. In this way, we form a set $T=\left\{x_{\alpha}: \alpha<\kappa\right\}$.

We obtain from Lemma 3.2 that $T$ is an approximable $\mathcal{W} \mathcal{A} \mathcal{P}_{0}(G)$-interpolation set. Since every infinite subset of $T$ is uniformly discrete and $C_{0}(G)$-interpolation sets must be relatively compact, and so finite (see the proof of Proposition 3.3 of [24]) any decomposition $T=\bigcup_{\eta<\kappa} T_{\eta}$ as a disjoint union of $\kappa$-many infinite subsets leaves us in position to apply Theorem 2.11 and finish the proof.

We deduce first that the quotient $\mathcal{W} \mathcal{A P}(G) /\left(\mathcal{A P}(G) \oplus C_{0}(G)\right)$ contains an isomorphic copy of $\ell_{\infty}(\kappa)$.

Corollary 4.2. Let $G$ be a non-compact locally compact E-group having an $E$-set $X$ with a compact covering number $\kappa$. Then the quotient space $\mathcal{W} \mathcal{A P}(G) /\left(\mathcal{A P}(G) \oplus C_{0}(G)\right)$ contains an isomorphic copy of $\ell_{\infty}(\kappa)$.

Proof. We only have to recall that $\mathcal{W} \mathcal{A P}(G)=\mathcal{A P}(G) \oplus \mathcal{W} \mathcal{A} \mathcal{P}_{0}(G)$, [42]. Therefore $\mathcal{W} \mathcal{A P}(G) /\left(\mathcal{A P}(G) \oplus C_{0}(G)\right)$ is isomorphic to $\mathcal{W} \mathcal{A P} \mathcal{P}_{0}(G) / C_{0}(G)$, and apply then Theorem 4.1. 
If we want to use our Theorem 2.11 to obtain a linear isometric copy of $\ell_{\infty}(\kappa)$ in the quotient $\mathcal{W} \mathcal{A P}(G) / \mathcal{A P}(G)$ we need first an approximable $\mathcal{W} \mathcal{A P}(G)$-interpolation set which is not an $\mathcal{A P}(G)$-interpolation set. If $G$, for instance, is discrete this means we need a translation-finite set that is not an $I_{0}$-set. Such sets can be easily found in $\mathbb{Z}$, the additive group of integers: $T=$ $\left\{3^{n}+n: n \in \mathbb{N}\right\} \cup\left\{3^{n}: n \in \mathbb{N}\right\}$ is such an example, see [30, Example 1.5.2] for a (simple) proof. For arbitrary discrete groups, an example as simple as that has escaped to us. A considerably more complicated construction can be used to obtain an approximable $\mathcal{W} \mathcal{A P}(G)$-interpolation set that is not a $\mathcal{B}(G)$-interpolation set, a fortiori not an $\mathcal{A P}(G)$-interpolation set when $G$ is an IN-group, a nilpotent group or a group with large enough centre, see Section 5 (note that the set $T$ above $i s$ a Sidon set, i.e., a $B(G)$-interpolation set).

Corollary 4.3. Let $G$ be a non-compact, locally compact E-group having an $E$-set $X$ with a compact covering number $\kappa$. Then the Banach space $\mathcal{W} \mathcal{A P}(G)$ contains a linear isometric copy $L$ of $\ell_{\infty}(\kappa)$ such that

$$
\frac{\|f\|}{2} \leq\left\|f+\mathcal{A P}(G) \oplus C_{0}(G)\right\|_{q} \leq\|f\| \quad \text { for every } \quad f \in L .
$$

In particular, the quotient space $\mathcal{W} \mathcal{A P}(G) /\left(\mathcal{A P}(G) \oplus C_{0}(G)\right)$ is non-separable.

Proof. Since $\mathcal{W A P}_{0}(G)$ is an ideal in the $C^{*}$-algebra $\mathcal{W} \mathcal{A P}(G)$, we see that every element in $G^{\mathcal{A P}}$ can be extended trivially on $\mathcal{W} \mathcal{A} \mathcal{P}_{0}(G)$. It follows that $\|f+g\| \geq\|g\|$ for every $f \in \mathcal{W A}_{\mathcal{A}}(G)$ and $g \in \mathcal{A P}(G)$. Therefore,

$$
\|f+g\| \geq \frac{1}{2}\|f\| \quad \text { for every } \quad f \in \mathcal{W} \mathcal{A} \mathcal{P}_{0}(G) \quad \text { and } \quad g \in \mathcal{A P}(G
$$

(use the previous inequality when $\frac{1}{2}\|f\| \leq\|g\|$, and the triangle inequality when $\left.\frac{1}{2}\|f\| \geq\|g\|\right)$. This gives for the quotient norms

$$
\left\|f+\mathcal{A P}(G) \oplus C_{0}(G)\right\|_{q} \geq \frac{1}{2}\left\|f+C_{0}(G)\right\|_{q} \quad \text { for every } \quad f \in \mathcal{W} \mathcal{A} \mathcal{P}_{0}(G) .
$$

Now Theorem 4.1 completes the proof since it gives an isometric copy $L$ of $\ell_{\infty}(\kappa)$ in $\mathcal{W A P}_{0}(G)$ such that $\|f\|=\left\|f+C_{0}(G)\right\|_{q}$ for every $f \in L$.

The results in this section will be considerably improved in the next section when $G$ is an $I N$-group or a nilpotent group.

\section{The Quotient of $\mathcal{W} \mathcal{A P}(G)$ By $\mathcal{B}(G)$}

The situation is much more delicate with $\mathcal{W} \mathcal{A P}(G) / \mathcal{B}(G)$. Already in the cases dealt with by Rudin in [49] and by Ramirez in [47] proving that $\mathcal{B}(G) \subsetneq \mathcal{W} \mathcal{A P}(G)$ the arguments were quite involved. Elaborating on the work by Rudin and Ramirez, Chou proved in [13] that the quotient space $\mathcal{W} \mathcal{A P}(G) / \mathcal{B}(G)$ contains a linear isometric copy of $\ell_{\infty}$ whenever $G$ is a noncompact, locally compact, $I N$-group or a nilpotent group. In all these papers the key argument consists in constructing a $t$-set that contains large squares. We follow here that thread and find copies of $\ell_{\infty}(\kappa)$ for $\kappa$ as large as possible in $\mathcal{W} \mathcal{A P}(G) / \mathcal{B}(G)$ by applying Theorem 2.11 . 
More precisely, we shall strengthen Chou's theorems by showing that there is a copy of $\ell_{\infty}(\kappa)$ in the quotient $\mathcal{W} \mathcal{A P}(G) / \mathcal{B}(G)$ when $G$ is either an IN-group or a nilpotent group and $\kappa=\kappa(G)$, and that, in general, for every locally compact group a copy of $\ell_{\infty}(\kappa(Z(G)))$ can be found in the quotient $\mathcal{W} \mathcal{A} \mathcal{P}(G) / \mathcal{B}(G)$.

The following technical lemma establishes that a group cannot be covered by $\beta$-cosets of finitely many different subgroups of index larger than $\beta$. This is similar to a theorem, known at least from the times of [45], in which only finitely many cosets are allowed.

Lemma 5.1. Let $G$ be any group with $|G|=\kappa$. Suppose that there is a finite collection $\left\{H_{1}, \ldots, H_{n}\right\}$ of subgroups of $G$ such that $G$ can be covered by $\beta<\kappa$ right-cosets of them, i.e., such that

$$
G=\bigcup_{j=1}^{n} \bigcup_{i \in I_{j}} H_{j} x_{i, j}, \quad \text { with }\left|I_{1}\right|+\cdots+\left|I_{n}\right|=\beta<\kappa .
$$

Then some of the subgroups $H_{j}$ has index at most $\beta$.

Proof. This is proved by induction on $n$. The theorem is obvious if $n=1$. Assume the theorem has been proved for unions of cosets of $n-1$ different subgroups and suppose

$$
G=\bigcup_{j=1}^{n} \bigcup_{i \in I_{j}} H_{j} x_{i, j}, \quad \text { with }\left|I_{1}\right|+\cdots+\left|I_{n}\right|=\beta<\kappa .
$$

If $\left|G: H_{n}\right|>\beta$, there is $x \in G$ such that $x \notin \bigcup_{i \in I_{n}} H_{n} x_{i, n}$. Since

$$
H_{n} x \cap H_{n} x_{i, n}=\emptyset \quad \text { for every } \quad i \in I_{n},
$$

we obtain

$$
H_{n} x \subseteq \bigcup_{j=1}^{n-1} \bigcup_{i \in I_{j}} H_{j} x_{i, j}
$$

and so

$$
H_{n}=\bigcup_{j=1}^{n-1} \bigcup_{i \in I_{j}} H_{j} x_{i, j} x^{-1} \cap H_{n}=\bigcup_{j=1}^{n-1} \bigcup_{i \in I_{j}}\left(H_{j} \cap H_{n}\right) y_{i, j},
$$

where the $y_{i, j}$ 's have been suitably chosen in $H_{n}$ (if $h_{j} x_{i, j} x^{-1}=y_{i, j}$ is in $H_{j} x_{i, j} x^{-1} \cap H_{n}$, then $\left.x_{i, j} x^{-1}=h_{j}^{-1} y_{i, j}\right)$. Applying our inductive hypothesis, we deduce that there is $j_{0}$ with $\left|H_{n}:\left(H_{n} \cap H_{j_{0}}\right)\right| \leq \beta$. (One may also proceed directly and replace $H_{n}$ from $(* *)$ in $(*)$, then apply the inductive hypothesis).

There is therefore a family $\left\{z_{s}: s \in S\right\} \subset H_{n}$ with $|S| \leq \beta$ such that $H_{n}=\bigcup_{s \in S}\left(H_{n} \cap H_{j_{0}}\right) z_{s}$ and we may replace (6) by

$$
G=\left(\bigcup_{j=1}^{n-1} \bigcup_{i \in I_{j}} H_{j} x_{i, j}\right) \bigcup\left(\bigcup_{i \in I_{n}} \bigcup_{s \in S} H_{j_{0}} z_{s} x_{i, n}\right) .
$$

Since this is a cover of $G$ by cosets of at most $n-1$ different subgroups of $G$ we deduce from our inductive hypothesis that some of the subgroups $H_{j}$, $1 \leq j \leq n-1$, has index at most $\beta$. 
Lemma 5.2. Let $G$ be a locally compact group containing a normal subgroup $H \subset G$. If $|G: H|=\kappa \geq \omega$, then $G$ contains a family $\left\{T_{\eta}: \eta<\kappa\right\}$ of subsets such that, putting $T=\bigcup_{\eta<\kappa} T_{\eta}$,

(i) $H t \cap H t^{\prime}=\emptyset$ for every $t \neq t^{\prime} \in T$.

(ii) $T_{\eta}$ contains large squares for every $\eta<\kappa$.

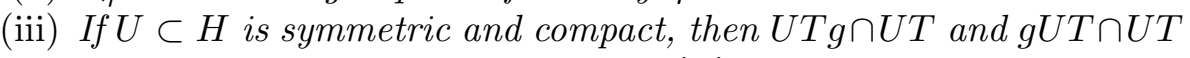
are relatively compact for every $g \notin \bigcup_{t \in T} t^{-1} U^{2} t$.

Proof. For each $\eta<\kappa$, let in this proof $\mathfrak{C}_{\eta}(H)$ denote the set

$$
\mathfrak{C}_{\eta}(H)=\{g H \in G / H:|\mathfrak{C r}(g H)| \leq \eta\},
$$

where $\mathfrak{C l}(g H)$ denotes the conjugacy class of $g H$ in $G / H$. If $A \subseteq G / H, \mathfrak{C l}(A)$ will stand for the set $\left\{\left(g^{-1} a g\right) H: g H \in G / H, a H \in A\right\} . \pi: G \rightarrow G / H$ will denote the canonical quotient mapping. For $n<\omega$, we define $\langle A\rangle_{n}$ to be the set of all products of at most $n$ elements in $A \cup A^{-1}$.

We will define for each $\eta<\kappa$ and $n<\omega$ two collections of finite sets

$$
C_{\eta, n}=\left\{x_{\eta, n, k}: 0 \leq k \leq n\right\} \quad \text { and } \quad D_{\eta, n}=\left\{y_{\eta, n, k}: 0 \leq k \leq n\right\} .
$$

These sets are defined recursively. First we order the set $\kappa \times \omega$ in the canonical way $([37,3.12])$ : For $\eta, \eta^{\prime}<\kappa$ and $n, n^{\prime}<\omega$, we define $(\eta, n)<$ $\left(\eta^{\prime}, n^{\prime}\right)$ if either $\max (\eta, n)<\max \left(\eta^{\prime}, n^{\prime}\right)$, or $\max (\eta, n)=\max \left(\eta^{\prime}, n^{\prime}\right)$ and $\eta<\eta^{\prime}$, or $\max (\eta, n)=\max \left(\eta^{\prime}, n^{\prime}\right), \eta=\eta^{\prime}$ and $n<n^{\prime}$. This way, the cardinal of the set $\left\{(\eta, n):(\eta, n)<\left(\eta_{0}, n_{0}\right)\right\}$ is less than $\kappa$ for every $\left(\eta_{0}, n_{0}\right) \in \kappa \times \omega$.

We now define $x_{0,0,0}=y_{0,0,0}=e$ and set $C_{0,0}=\left\{x_{0,0,0}\right\}, D_{0,0}=\left\{y_{0,0,0}\right\}$.

Assume that the sets $C_{\gamma, j}$ and $D_{\gamma, j}$ have been defined for $(\gamma, j)<(\eta, n)$. We now define the set $C_{\eta, n}$.

To that end we need define $R_{\eta, n, 0}=\bigcup_{(\gamma, j)<(\eta, n)}\left(\pi\left(C_{\gamma, j}\right) \cup \pi\left(D_{\gamma, j}\right)\right)$ and consider a cardinal number $f(\eta, n, 0)$ such that

$$
\kappa>f(\eta, n, 0)>(n+1)^{2}\left|R_{\eta, n, 0}\right|^{4}+\left(\left|R_{\eta, n, 0}\right|+n+1\right)^{8} .
$$

Note that, in particular, $f(\eta, n, 0)$ is finite when $\kappa=\omega$.

We then choose $x_{\eta, n, 0}$ such that

$$
\pi\left(x_{\eta, n, 0}\right) \notin\left\langle R_{\eta, n, 0}, \mathfrak{C l}\left(R_{\eta, n, 0} \cap \mathfrak{C}_{f(\eta, n, 0)}\right)\right\rangle_{8} .
$$

The choice of this element will be possible because $\left|\left\langle R_{\eta, n, 0}\right\rangle_{8}\right| \leq f(\eta, n, 0)<$ $\kappa=|G: H|$ and conjugacy classes of elements of $\mathfrak{C}_{f(\eta, n, 0)}$ do not have, by definition, more than $f(\eta, n, 0)$-elements.

Suppose now that $x_{\eta, n, l}$ has been defined for $0 \leq l<k$. The element $x_{\eta, n, k}$ is then defined similarly. We first define

$$
R_{\eta, n, k}=\bigcup_{(\gamma, j)<(\eta, n)}\left(\pi\left(C_{\gamma, j}\right) \cup \pi\left(D_{\gamma, j}\right)\right) \bigcup\left\{\pi\left(x_{\eta, n, 0}\right), \ldots, \pi\left(x_{\eta, n, k-1}\right)\right\}
$$

and $f(\eta, n, k)$ such that

$$
\kappa>f(\eta, n, k)>(n+1)^{2}\left|R_{\eta, n, k}\right|^{4}+\left(\left|R_{\eta, n, k}\right|+2 n+1\right)^{8} .
$$


Then $x_{\eta, n, k}$ is obtained as above:

$$
\pi\left(x_{\eta, n, k}\right) \notin\left\langle R_{\eta, n, k}, \mathfrak{C l}\left(R_{\eta, n, k} \cap \mathfrak{C}_{f(\eta, n, 0)}\right)\right\rangle_{8} .
$$

Once the sets $C_{\gamma, j}$ have been defined for $(\gamma, j) \leq(\eta, n)$ and $D_{\gamma, j}$ have been defined for $(\gamma, j)<(\eta, n)$, we define the set $D_{\eta, n}$.

The process starts as in the case of $C_{\eta, n}$, in this case the elements of this latter set must also be avoided. We thus start by defining the sets:

$$
\begin{aligned}
& S_{\eta, n, 0}=\left(\bigcup_{(\gamma, j) \leq(\eta, n)} \pi\left(C_{\gamma, j}\right)\right) \bigcup\left(\bigcup_{(\gamma, j)<(\eta, n)} \pi\left(D_{\gamma, j}\right)\right) \text { and, for } 1 \leq k \leq n, \\
& S_{\eta, n, k}=\left(\bigcup_{(\gamma, j) \leq(\eta, n)} \pi\left(C_{\gamma, j}\right)\right) \bigcup\left(\bigcup_{(\gamma, j)<(\eta, n)} \pi\left(D_{\beta, i}\right)\right) \bigcup\left\{\pi\left(y_{\eta, n, 0}\right), \ldots, \pi\left(y_{\eta, n, k-1}\right)\right\}
\end{aligned}
$$

We use the following claim to define $y_{\eta, n, k}$.

Claim 1: There is $y_{\eta, n, k} \in G$ such that

$$
\pi\left(y_{\eta, n, k}\right) \notin\left\langle S_{\eta, n, k}\right\rangle_{8}
$$

and

$$
C_{\eta, n} C_{\eta, n}^{-1} \bigcap y_{\eta, n, k} \pi^{-1}\left(\left\langle R_{\eta, n, k}\right\rangle_{4}\right) y_{\eta, n, k}^{-1}=\{e\}
$$

We first enumerate $\left(C_{\eta, n} C_{\eta, n}^{-1} \backslash\{e\}\right) \bigcap \pi^{-1}\left(\left\langle\mathfrak{C l}\left(R_{\eta, n, k}\right)\right\rangle_{4}\right)$ as $\left\{a_{1}, \ldots, a_{l}\right\}$. Let then

$$
R_{j}=\pi^{-1}\left(\left\langle R_{\eta, n, k}\right\rangle_{4} \cap \mathfrak{C l}\left(\pi\left(a_{j}\right)\right)\right)
$$

and choose, for each $j, 1 \leq j \leq l$, and each $r \in R_{j}$ an element $y_{j, r} \in G$ and an element $h_{j, r} \in H$ with

$$
r=y_{j, r}^{-1} a_{j} h_{j, r} y_{j, r} .
$$

Suppose now that no $y \in G$ can be found so that conditions (11) and (12) are satisfied. In that case some $R_{j}$ must be non-empty and, indeed,

$$
G=\left\langle S_{\eta, n, k}\right\rangle_{8} \bigcup\left(\bigcup_{j=1}^{l} \bigcup_{r \in R_{j}} L_{j, r}\right),
$$

where

$$
L_{j, r}=\left\{g \in G: r=g^{-1} a_{j} g\right\} .
$$

Observe now that that $\pi\left(L_{j, r}\right) \subseteq C_{G / H}\left(\pi\left(a_{j}\right)\right) \pi\left(y_{j, r}\right)$, where

$$
C_{G / H}\left(\pi\left(a_{j}\right)\right)=\left\{\pi(g) \in G / H: \pi\left(g a_{j}\right)=\pi\left(a_{j} g\right)\right\}
$$

is the centralizer of $\pi\left(a_{j}\right)$. Therefore,

$$
G / H=\left\langle S_{\eta, n, k}\right\rangle_{8} \bigcup\left(\bigcup_{j=1}^{l} \bigcup_{r \in R_{j}} C_{G / H}\left(\pi\left(a_{j}\right)\right) \pi\left(y_{j, r}\right)\right) .
$$

Now, $\left|S_{\eta, n, 0}\right| \leq\left|R_{\eta, n, 0}\right|+n+1$ and $\left|S_{\eta, n, k}\right| \leq\left|R_{\eta, n, 0}\right|+2 n+1$, for $1 \leq k \leq n$. We therefore deduce from (7) and (9) that $(n+1)^{2}\left|\left\langle R_{\eta, n, k}\right\rangle_{4}\right|+\left|\left\langle S_{\eta, n, k}\right\rangle_{8}\right|<f(\eta, n, k)$ for $0 \leq k \leq n$. 
If the elements of the set $\left\langle S_{\eta, n, k}\right\rangle_{8}$ are viewed as cosets of the trivial subgroup $\left\{e_{G / H}\right\}$, we see in (13) that $G / H$ can be decomposed as a union of less than $(n+1)^{2}\left|\left\langle R_{\eta, n, k}\right\rangle_{4}\right|+\left|\left\langle S_{\eta, n, k}\right\rangle_{8}\right|$ cosets. We deduce from Lemma 5.1 that some of them must correspond to a subgroup of index at most $f(\eta, n, k)$. Thus, there is $j, 1 \leq j \leq l$ such that $\left|G / H: C_{G / H}\left(\pi\left(a_{j}\right)\right)\right|=\left|\mathfrak{C l}\left(\pi\left(a_{j}\right)\right)\right|<$ $f(\eta, n, k)$. We conclude that $\pi\left(a_{j}\right) \in \mathfrak{C}_{f(\eta, n, k)}$.

Since $\pi\left(a_{j}\right) \cap \mathfrak{C l}\left(R_{\eta, n, k}\right) \neq \emptyset$, we find that $a_{j} \in \pi^{-1}\left(\mathfrak{C l}\left(R_{n, \eta, k} \cap \mathfrak{C}_{f(\eta, n, k)}\right)\right)$. If $a_{j}=x_{\eta, n, k_{1}}^{-1} x_{\eta, n, k_{2}}$ and $k_{2}>k_{1}$, this goes against condition (10) in the choice of $x_{\eta, n, k_{2}}$ and finishes the proof of the claim.

We have therefore constructed two families

$C_{\eta, n}=\left\{x_{\eta, n, k}: 0 \leq k \leq n\right\} \quad$ and $D_{\eta, n}=\left\{y_{\eta, n, k}: 0 \leq k \leq n\right\}, \quad(\eta, n) \in \kappa \times \omega$, with properties (10), (11) and (12). We now check that the sets

$$
T_{\eta}=\bigcup_{n<\omega}\left(D_{\eta, n} C_{\eta, n}\right), \quad \eta<\kappa
$$

satisfy the desired properties.

First of all we see that $\left|D_{\eta, n} C_{\eta, n}\right|=(n+1)^{2}$. If this were not the case, there would be $1 \leq k_{1}, k_{2}, k_{3}, k_{4} \leq n$ with $k_{1} \neq k_{3}$ and $k_{2}<k_{4}$ such that $y_{\eta, n, k_{1}} x_{\eta, n, k_{2}}=y_{\eta, n, k_{3}} x_{\eta, n, k_{4}}$. But then $y_{\eta, n, k_{4}} \in\left\langle C_{\eta, n}, y_{\eta, n, k_{2}}\right\rangle_{2}$ which goes against our choice of the elements in $D_{\eta, n}$. Therefore, $T_{\eta}$ contains $n$-squares for every $n$.

In order to prove the last statement, we take $U$ a compact subset of $H$ and $g \notin U^{2}$. Choose $t_{1}=y_{\eta_{1}, n_{1}, k_{1}} x_{\eta_{1}, n_{1}, k_{1}^{\prime}}$ and $t_{2}=y_{\eta_{2}, n_{2}, k_{2}} x_{\eta_{2}, n_{2}, k_{2}^{\prime}}, u_{1}, u_{2} \in U$, with

$$
g u_{1} t_{1}=u_{2} t_{2} \in g U T \cap U T .
$$

We order the 3 -tuples $\left(\eta_{i}, n_{i}, k_{i}\right)$ lexicographically with respect to the last entry, that is $\left(\eta_{i}, n_{i}, k_{i}\right)>\left(\eta_{i}^{\prime}, n_{i}^{\prime}, k_{i}^{\prime}\right)$ if either $\left(\eta_{i}, n_{i}\right)>\left(\eta_{i}^{\prime}, n_{i}^{\prime}\right)$ or $\left(\eta_{i}, n_{i}\right)=$ $\left(\eta_{i}^{\prime}, n_{i}^{\prime}\right)$ and $k_{i}>k_{i}^{\prime}$.

Assume that $\left(\eta_{2}, n_{2}\right) \geq\left(\eta_{1}, n_{1}\right)$.

Let now $g u_{3} t_{3}=u_{4} t_{4}$ with $t_{3}=y_{\eta_{3}, n_{3}, k_{3}} x_{\eta_{3}, n_{3}, k_{3}^{\prime}}$ and $t_{4}=y_{\eta_{4}, n_{4}, k_{4}} x_{\eta_{4}, n_{4}, k_{4}^{\prime}}$, $u_{3}, u_{4} \in U$ be any other element of $g U T \cap U T$.

Then

$$
\begin{aligned}
g & =u_{4} y_{\eta_{4}, n_{4}, k_{4}} x_{\eta_{4}, n_{4}, k_{4}^{\prime}} x_{\eta_{3}, n_{3}, k_{3}^{\prime}}^{-1} y_{\eta_{3}, n_{3}, k_{3}}^{-1} u_{3}^{-1} \\
& =u_{2} y_{\eta_{2}, n_{2}, k_{2}} x_{\eta_{2}, n_{2}, k_{2}^{\prime}} x_{\eta_{1}, n_{1}, k_{1}^{\prime}}^{-1} y_{\eta_{1}, n_{1}, k_{1}}^{-1} u_{1}^{-1} .
\end{aligned}
$$

Let $\left(\eta_{i_{1}}, n_{i_{1}}, k_{i_{1}}\right)=\max \left\{\left(\eta_{i}, n_{i}, k_{i}\right): 1 \leq i \leq 4\right\}$. There must then be $i_{2}$, $1 \leq i_{2} \leq 4, i_{2} \neq i_{1}$, with $\eta_{i_{1}}=\eta_{i_{2}}, n_{i_{1}}=n_{i_{2}}$ and $y_{\eta_{i_{1}}, n_{i_{1}}, k_{i_{1}}}=y_{\eta_{i_{2}}, n_{i_{2}}, k_{i_{2}}}$, for otherwise $y_{\eta_{i_{1}}, n_{i_{1}}, k_{i_{1}}} \in\left\langle S_{\eta_{i_{1}}, n_{i_{1}}, k_{i_{1}}}\right\rangle_{7}$.

Claim 2: It is not possible that $\left(\eta_{4}, n_{4}\right)=\left(\eta_{3}, n_{3}\right)>\left(\eta_{2}, n_{2}\right)$.

Should this be the case, then $y_{\eta_{4}, n_{4}, k_{4}}=y_{\eta_{3}, n_{3}, k_{3}}$ and

$$
x_{\eta_{3}, n_{3}, k_{4}^{\prime}} x_{\eta_{3}, n_{3}, k_{3}^{\prime}}^{-1} \in y_{\eta_{3}, n_{3}, k_{3}}^{-1}\left(y_{\eta_{2}, n_{2}, k_{2}} x_{\eta_{2}, n_{2}, k_{2}^{\prime}} x_{\eta_{1}, n_{1}, k_{1}^{\prime}}^{-1} y_{\eta_{1}, n_{1}, k_{1}}^{-1}\right) y_{\eta_{3}, n_{3}, k_{3}} H .
$$

It follows from our condition (12) in the choice of $y_{\eta_{3}, n_{3}, k_{3}}$ (Claim 1, page 21 ) that $x_{\eta_{3}, n_{3}, k_{4}^{\prime}}=x_{\eta_{3}, n_{3}, k_{3}^{\prime}}^{-1}$ but this is only possible if $g \in U^{2}$ (take into account hat $g u_{3} t_{3}=u_{4} t_{4}$ and that $\left.y_{\eta_{4}, n_{4}, k_{4}}=y_{\eta_{3}, n_{3}, k_{3}}\right)$, and the claim is proved. 
The same argument shows that it is not possible that $\left(\eta_{1}, n_{1}\right)=\left(\eta_{2}, n_{2}\right)>$ $\left(\eta_{i}, n_{i}\right)$, with $i=3,4$.

We deduce that either $\left(\eta_{4}, n_{4}\right)=\left(\eta_{2}, n_{2}\right)$ or $\left(\eta_{3}, n_{3}\right)=\left(\eta_{2}, n_{2}\right)$. But, since the element $g u_{3} t_{3}=u_{4} t_{4}$ was chosen arbitrarily in $g U T \cap U T$, it follows that

$$
g U T \cap U T \subseteq\left(g U D_{\eta_{2}, n_{2}} C_{\eta_{2}, n_{2}}\right) \cup\left(U D_{\eta_{2}, n_{2}} C_{\eta_{2}, n_{2}}\right),
$$

and this is a relatively compact set.

We now check that $U T g \cap U T$ is compact. Choose $t_{1}=y_{\eta_{1}, n_{1}, k_{1}} x_{\eta_{1}, n_{1}, k_{1}^{\prime}}$ and $t_{2}=y_{\eta_{2}, n_{2}, k_{2}} x_{\eta_{2}, n_{2}, k_{2}^{\prime}}, u_{1}, u_{2} \in U$ with

$$
u_{1} t_{1} g=u_{2} t_{2} \in U T g \cap U T .
$$

Let

$$
u_{3} y_{\eta_{3}, n_{3}, k_{3}} x_{\eta_{3}, n_{3}, k_{3}^{\prime}} g=u_{4} y_{\eta_{4}, n_{4}, k_{4}} x_{\eta_{4}, n_{4}, k_{4}^{\prime}}, \quad u_{3}, u_{4} \in U
$$

be any other element of $U T g \cap U T$ with $\left(\eta_{3}, n_{3}\right) \geq\left(\eta_{4}, n_{4}\right)$. We have that

$$
\begin{aligned}
g & =t_{1}^{-1} u_{1}^{-1} u_{2} t_{2} \\
& =x_{\eta_{3}, n_{3}, k_{3}^{\prime}}^{-1} y_{\eta_{3}, n_{3}, k_{3}}^{-1} u_{3}^{-1} u_{4} y_{\eta_{4}, n_{4}, k_{4}} x_{\eta_{4}, n_{4}, k_{4}^{\prime}} \in x_{\eta_{3}, n_{3}, k_{3}^{\prime}}^{-1} y_{\eta_{3}, n_{3}, k_{3}}^{-1} y_{\eta_{4}, n_{4}, k_{4}} x_{\eta_{4}, n_{4}, k_{4}^{\prime}} H .
\end{aligned}
$$

As in the preceding case we assume that $\left(\eta_{2}, n_{2}\right) \geq\left(\eta_{1}, n_{1}\right)$ and it is enough to see that neither $\left(\eta_{4}, n_{4}\right)=\left(\eta_{3}, n_{3}\right)>\left(\eta_{2}, n_{2}\right)$, nor $\left(\eta_{2}, n_{2}\right)=$ $\left(\eta_{1}, n_{1}\right)>\left(\eta_{3}, n_{3}\right)$.

If $\left(\eta_{4}, n_{4}\right)=\left(\eta_{3}, n_{3}\right)>\left(\eta_{2}, n_{2}\right)$, then necessarily $\left(\eta_{4}, n_{4}, k_{4}\right)=\left(\eta_{3}, n_{3}, k_{3}\right)$ and $t_{1}^{-1} u_{1}^{-1} u_{2} t_{2} \in x_{\eta_{3}, n_{3}, k_{3}^{\prime}}^{-1} x_{\eta_{3}, n_{3}, k_{4}^{\prime}} H$. If $k_{3}^{\prime}>k_{4}^{\prime}$, then $x_{\eta_{3}, n_{3}, k_{3}^{\prime}} \in\left\langle R_{\eta_{3}, n_{3}, k_{3}^{\prime}}\right\rangle_{5}$, against the election of $x_{\eta_{3}, n_{3}, k_{3}^{\prime}}$. But $k_{3}^{\prime}=k_{4}^{\prime}$, implies that $t_{3}=t_{4}$ (recall that $\left.\left(\eta_{4}, n_{4}, k_{4}\right)=\left(\eta_{3}, n_{3}, k_{3}\right)\right)$ and $g \in t_{3}^{-1} U^{2} t_{3}$. We rule out analogously the possibility $\left(\eta_{1}, n_{1}\right)=\left(\eta_{2}, n_{2}\right)$ and argue as above to prove that

$$
U T g \cap U T \subseteq\left(U D_{\eta_{2}, n_{2}} C_{\eta_{2}, n_{2}}\right) g \cup\left(U D_{\eta_{2}, n_{2}} C_{\eta_{2}, n_{2}}\right),
$$

and conclude that $U T g \cap U T$ is relatively compact.

Corollary 5.3. Let $G$ be a locally compact group, $H$ a subgroup of $G$ and $N_{G}(H)=\{g \in G: g H=H g\}$ be the normalizer of $H$ in $G$. If $\left|N_{G}(H): H\right|=\kappa \geq \omega$, then $N_{G}(H)$ contains a family $\left\{T_{\eta}: \eta<\kappa\right\}$ of subsets such that

(i) if $T=\bigcup_{\eta<\kappa} T_{\eta}$, then $H t \cap H t^{\prime}=\emptyset$ for every $t \neq t^{\prime} \in T$;

(ii) $T_{\eta}$ contains large squares for every $\eta<\kappa$;

(iii) if $T=\bigcup_{\eta<\kappa} T_{\eta}$ and $U \subset H$ is compact, then $U T g \cap U T$ and $g U T \cap$ $U T$ are relatively compact for every $g \notin \bigcup_{t \in T} t^{-1} U^{2} t$.

Proof. Since $H$ is a normal subgroup of $N_{G}(H)$, we can apply Lemma 5.2 to $N_{G}(H)$. Statements (i) and (ii) remain the same if $N_{G}(H)$ is replaced by $G$. As for Statement (iii), one notices that $g U T \cap U T \neq \emptyset$ and $U T g \cap U T \neq \emptyset$ both imply that $g \in N_{G}(H)$, and so this statement follows also from Lemma 5.2 .

We obtain a first consequence for groups with large center.

Theorem 5.4. Let $G$ be a locally compact group, $Z(G)$ be the algebraic center of $G$ and put $\kappa=\kappa(Z(G))$. If $\kappa \neq 1$, then there is always a linear isometry $\Psi: \ell_{\infty}(\kappa) \rightarrow \mathcal{W} \mathcal{A P}(G) / \mathcal{B}(G)$. 
Proof. The center $Z(G)$, as every locally compact Abelian group, always contains an open subgroup $G_{0}$ topologically isomorphic to $\mathbb{R}^{n} \times K$, with $K$ compact. Therefore, $G$ must contain two subgroups $H_{1} \subset H_{2} \subseteq Z(G)$ with $H_{1}$ open in $H_{2}$ and $\left|H_{2}: H_{1}\right|=\kappa$. Indeed, if $\left|Z(G): G_{0}\right| \geq \omega$, then we take $H_{2}=Z(G)$ and $H_{1}=G_{0}$. If $G_{0}$ has finite index and $Z(G)$ is not compact, then $\kappa=\omega$ and so we may take $H_{2}=\mathbb{Z}$ and $H_{1}=\{e\}$.

Let $\left\{T_{\eta}: \eta<\kappa\right\}$ be the family of subsets of $H_{2}$ provided by Lemma 5.2. By Theorem 3.5, none of them is a $\mathcal{B}(G)$-interpolation set. If $T=\bigcup_{\eta<\kappa} T_{\eta}$ and $U$ is a compact neighbourhood of the identity in $H_{2}$ with $U \subset H_{1}$, then, since $H_{2}$ is commutative, $U T$ is a t-set in $H_{2}$. By Lemma 3.3, we can find a compact neighbourhood $V$ of the identity in $G$, such that $V T$ is a t-set in $G$. If $V$ is chosen so that $V^{4} \cap H_{2} \subset H_{1}$ (remember $H_{1}$ is open in $H_{2}$ ), then $T$ is $V^{2}$-uniformly discrete, and by Lemma $3.2, T$ is an approximable $\mathcal{W} \mathcal{A P}(G)$-interpolation set.

It suffices now to apply Theorem 2.11.

Lemma 5.2 can be readily applied to discrete groups. To further expand its applicability we follow the usual path applying well-known structure theorems. The following Lemma for instance is the analogue of Lemma 4.4 of [13].

Lemma 5.5. Let $G$ be a locally compact group and let $N$ be a closed subgroup of $G$.

(i) If $N$ is normal, the quotient map $\pi: G \rightarrow G / N$ induces linear isometries

$$
\begin{aligned}
& \Pi: \mathcal{W} \mathcal{A P}(G / N) / \mathcal{B}(G / N) \rightarrow \mathcal{W} \mathcal{A P}(G) / \mathcal{B}(G) \text { and } \\
& \Pi_{0}: \mathcal{W A P}_{0}(G / N) / \mathcal{B}_{0}(G / N) \rightarrow \mathcal{W} \mathcal{A} \mathcal{P}_{0}(G) / \mathcal{B}_{0}(G) .
\end{aligned}
$$

(ii) If $N$ is open, there are linear isometries

$$
\begin{aligned}
& \Psi: \mathcal{W} \mathcal{A P}(N) / \mathcal{B}(N) \rightarrow \mathcal{W} \mathcal{A P}(G) / \mathcal{B}(G) \text { and } \\
& \Psi_{0}: \mathcal{W A P}_{0}(N) / \mathcal{B}_{0}(N) \rightarrow \mathcal{W} \mathcal{A P}_{0}(G) / \mathcal{B}_{0}(G) .
\end{aligned}
$$

Proof. We first prove (i). The map $\phi \mapsto \phi \circ \pi$ clearly defines a linear isometry $\tilde{\pi}: \mathcal{W} \mathcal{A P}(G / N) \rightarrow \mathcal{W} \mathcal{A P}(G)$. By [11, Theorem], we have

$$
\tilde{\pi}(\mathcal{B}(G / N))=\tilde{\pi}(\mathcal{E B}(G / N)) \cap \mathcal{B}(G) .
$$

By [9], we have

$$
\tilde{\pi}(\mathcal{W} \mathcal{A} \mathcal{P}(G / N))=\tilde{\pi}(\mathcal{C B}(G / N)) \cap \mathcal{W} \mathcal{A P}(G) .
$$

Since $\mathcal{B}(G) \subseteq \mathcal{W} \mathcal{A P}(G)$, we see that

$$
\tilde{\pi}(\mathcal{B}(G / N))=\tilde{\pi}(\mathcal{C B}(G / N)) \cap \mathcal{W} \mathcal{A P}(G) \cap \mathcal{B}(G)=\tilde{\pi}(\mathcal{W} \mathcal{A P}(G / N)) \cap \mathcal{B}(G)
$$

so that $\tilde{\pi}$ induces a linear isomorphism

$$
\Pi: \mathcal{W} \mathcal{A P}(G / N) / \mathcal{B}(G / N) \rightarrow \mathcal{W} \mathcal{A P}(G) / \mathcal{B}(G),
$$

given by

$$
\Pi(\phi+\mathcal{B}(G / N))=\tilde{\pi}(\phi)+\mathcal{B}(G) .
$$


We check that $\Pi$ is an isometry. If $\phi \in \mathcal{W} \mathcal{A P}(G / N)$,

$$
\begin{aligned}
\|\Pi(\phi+\mathcal{B}(G / N))\| & =\|\tilde{\pi}(\phi)+\mathcal{B}(G)\| \\
& =\inf \{\|\tilde{\pi}(\phi)+\psi\|: \psi \in \mathcal{B}(G)\} \\
& \leq \inf \{\|\tilde{\pi}(\phi+\psi)\|: \psi \in \mathcal{B}(G / N)\} \\
& =\inf \{\|\phi+\psi\|: \psi \in \mathcal{B}(G / N)\}=\|\phi+\mathcal{B}(G / N)\| .
\end{aligned}
$$

For the reverse inequality, we follow the path of Lemma 2.3 of [12] and consider the invariant mean $\mu_{N}$ on $\mathcal{W} \mathcal{A P}(N)$. For $\phi \in \mathcal{W} \mathcal{A P}(G)$, we define the function $\phi^{N}: G \rightarrow \mathbb{C}$ by

$$
\phi^{N}(g)=\mu_{N}\left(\phi_{g}\right), \quad \text { where } \phi_{g}(h)=\phi(g h) .
$$

By invariance of $\mu_{N}$, the function $\phi^{N}$ is constant on the cosets of $N$ and therefore induces a continuous function on $G / N$. Clearly, $\left\|\phi^{N}\right\|_{G / N} \leq\|\phi\|_{G}$.

Now Lemma 2.3 of [12] proves in fact that $\phi^{N} \in \mathcal{W} \mathcal{A P}(G / N)$. Moreover, by first considering positive-definite functions, it is also easily checked that $\psi^{N} \in \mathcal{B}(G / N)$ for every $\psi \in \mathcal{B}(G)$.

Note as well that for $\phi \in \mathcal{W} \mathcal{A P}(G / N)$, we have $\tilde{\pi}(\phi)^{N}=\phi$.

Now if $\phi \in \mathcal{W} \mathcal{A P}(G / N)$ and $\psi \in \mathcal{B}(G)$,

$$
\begin{aligned}
\|\tilde{\pi}(\phi)+\psi\| & \geq\left\|(\tilde{\pi}(\phi)+\psi)^{N}\right\| \\
& =\left\|\tilde{\pi}(\phi)^{N}+\psi^{N}\right\| \\
& =\left\|\phi+\psi^{N}\right\| \\
& \geq\|\phi+\mathcal{B}(G / N)\| .
\end{aligned}
$$

And the remaining inequality

$$
\|\Pi(\phi+\mathcal{B}(G / N))\|=\|\tilde{\pi}(\phi)+\mathcal{B}(G)\| \geq\|\phi+\mathcal{B}(G / N)\|
$$

follows.

We prove now the analogue statements for $\mathcal{W A}_{\mathcal{A}}(G)$ and $\mathcal{B}_{0}(G)$. We check first that $\tilde{\pi}$ maps $\mathcal{W A P}_{0}(G / N)$ into $\mathcal{W A P}_{0}(G)$ and $\mathcal{B}_{0}(G / N)$ into $\mathcal{B}_{0}(G)$. Consider the adjoint of $\tilde{\pi}$, this is the map given by

$$
\tilde{\pi}^{*}: \mathcal{W} \mathcal{A P}(G)^{*} \rightarrow \mathcal{W} \mathcal{A P}(G / N)^{*}, \quad \tilde{\pi}^{*}(\nu)=\nu \circ \tilde{\pi} .
$$

Note that if $\mu \in \mathcal{W} \mathcal{A P}(G)^{*}$ is invariant then $\tilde{\pi}^{*}(\mu) \in \mathcal{W} \mathcal{A P}(G / N)^{*}$ is invariant. To see this, let $\bar{s}=\pi(s) \in G / N$ and $f \in \mathcal{W} \mathcal{A P}(G / N)$ and note that $\tilde{\pi}\left(f_{\bar{s}}\right)=(\tilde{\pi}(f))_{s}$, and so

$$
\tilde{\pi}^{*}(\mu)\left(f_{\bar{s}}\right)=\mu\left(\tilde{\pi}\left(f_{\bar{s}}\right)=\mu\left((\tilde{\pi}(f))_{s}\right)=\mu(\tilde{\pi}(f))=\tilde{\pi}^{*}(\mu)(f) .\right.
$$

Thus, $\tilde{\pi}^{*}(\mu)$ is invariant on $\mathcal{W} \mathcal{A} \mathcal{P}(G / N)$.

Let now $f \in \mathcal{W A P}_{0}(G / N)$ and $\mu$ be the invariant mean on $\mathcal{W} \mathcal{A P}(G)$. Then $\tilde{\pi}(f) \in \mathcal{W} \mathcal{A P}(G)$ and

$$
\mu(|\tilde{\pi}(f)|)=\mu(|f \circ \pi|)=\mu(|f| \circ \pi)=\tilde{\pi}^{*}(\mu)(|f|)=0 .
$$

Thus, $\tilde{\pi}(f) \in \mathcal{W A P}_{0}(G)$. To see that $\tilde{\pi}(f) \in \mathcal{B}_{0}(G)$ when $f \in \mathcal{B}_{0}(G / N)$, we argue in a similar way using the fact that $\tilde{\pi}(f) \in \mathcal{B}(G)$ by [12, Theorem]. Accordingly,

$$
\tilde{\pi}\left(\mathcal{B}_{0}(G / N)\right) \subseteq \tilde{\pi}\left(\mathcal{W} \mathcal{A} \mathcal{P}_{0}(G / N)\right) \cap \mathcal{B}_{0}(G)
$$


The reverse inclusion is checked as follows. If $f \in \tilde{\pi}\left(\mathcal{W A P}_{0}(G / N)\right) \cap$ $\mathcal{B}_{0}(G)$, then by (14) $f$ is clearly in $\tilde{\pi}(\mathcal{B}(G / N))$. So let $g \in \mathcal{B}(G / N)$ with $f=\tilde{\pi}(g)$. We only need to make sure that $\tilde{\pi}^{*}(\mu)(|g|)=0$. But this is also clear from the following identity.

$$
\tilde{\pi}^{*}(\mu)(|g|=\mu(\tilde{\pi}(|g|))=\mu(|g| \circ \pi)=\mu(|g \circ \pi|)=\mu(|\tilde{\pi}(g)|)=\mu(|f|) .
$$

Thus, we obtain the analogue of (14)

$$
\tilde{\pi}\left(\mathcal{B}_{0}(G / N)\right)=\tilde{\pi}\left(\mathcal{W} \mathcal{A} \mathcal{P}_{0}(G / N)\right) \cap \mathcal{B}_{0}(G)
$$

so that $\tilde{\pi}$ induces a linear isomorphism

$$
\Pi_{0}: \mathcal{W} \mathcal{A} \mathcal{P}_{0}(G / N) / \mathcal{B}_{0}(G / N) \rightarrow \mathcal{W} \mathcal{A} \mathcal{P}_{0}(G) / \mathcal{B}_{0}(G),
$$

given by

$$
\Pi_{0}\left(\phi+\mathcal{B}_{0}(G / N)\right)=\tilde{\pi}(\phi)+\mathcal{B}_{0}(G) .
$$

To check that $\Pi_{0}$ is an isometry, we proceed precisely as for $\Pi$.

For the proof of (ii), we associate to each $\phi \in \mathcal{W} \mathcal{A P}(N)$ the function

$$
\phi_{N}(g)=\phi(g) \quad \text { if } \quad g \in N \quad \text { and } \quad \phi_{N}(g)=0 \quad \text { if } \quad g \notin N .
$$

Then $\phi_{N}$ is in $\mathcal{W} \mathcal{A P}(G)$ by [43, Lemma 5.4], [9, Theorem 3.14] or [10, Lemma 2.4]. If $\phi$ happens to be in $B(N)$ then $\phi_{N} \in B(G)$ by [35, page 280] or [13, Lemma 4.1] and this obviously extends to $\mathcal{B}(N)$ and $\mathcal{B}(G)$.

Define then $\Psi: \mathcal{W} \mathcal{A P}(N) / \mathcal{B}(N) \rightarrow \mathcal{W} \mathcal{A P}(G) / \mathcal{B}(G)$ by

$$
\Psi(\phi+\mathcal{B}(N))=\phi_{N}+\mathcal{B}(G) .
$$

It is easy to check that $\Psi$ is a linear isometry.

To prove the second statement of (ii), note that the extension of $\phi_{N}$ defined in (16) is clearly in $\mathcal{W A P}_{0}(G)$ if $\phi \in \mathcal{W} \mathcal{A} \mathcal{P}_{0}(N)$, and in $\mathcal{B}_{0}(G)$ if $\phi \in \mathcal{B}_{0}(N)$. It is again straightforward to verify that

$\Psi_{0}: \mathcal{W} \mathcal{A} \mathcal{P}_{0}(N) / \mathcal{B}_{0}(N) \rightarrow \mathcal{W} \mathcal{A} \mathcal{P}_{0}(G) / \mathcal{B}_{0}(G), \quad \Psi_{0}\left(\phi+\mathcal{B}_{0}(N)\right)=\phi_{N}+\mathcal{B}_{0}(G)$ is the required linear isometry.

We reach finally our main results.

Theorem 5.6. Let $G$ be a a non-compact, locally compact, $I N$-group and put $\kappa=\kappa(G)$. Then there is a linear isometry $\Psi: \ell_{\infty}(\kappa) \rightarrow \mathcal{W} \mathcal{A P}(G) / \mathcal{B}(G)$.

Proof. By Theorem 2.13 of [33], there is an open normal subgroup $N$ of $G$ that contains a compact normal subgroup $K$ with $N / K$ Abelian.

Suppose first that $\kappa(N)=\kappa$. Then $\kappa=\kappa(N / K)$. By Theorem 5.4, there is a linear isometric copy of $\ell_{\infty}(\kappa)$ in $\mathcal{W} \mathcal{A P}(N / K) / \mathcal{B}(N / K)$. We apply then (i) and (ii) of Lemma 5.5 to obtain a linear isometric copy of $\ell_{\infty}(\kappa)$ in $\mathcal{W} \mathcal{A} \mathcal{P}(G) / \mathcal{B}(G)$.

If $\kappa(N)<\kappa$, it follows that $\kappa=|G: N|$. We apply Lemma 5.2 to the discrete group $G / N$. Let $\left\{T_{\eta}: \eta<\kappa\right\}$ be the collection of subsets obtained in that Lemma (in this case the subgroup $H$ of that Lemma is trivial) and let $T=\bigcup_{\eta<\kappa} T_{\eta}$. By (iii) in that Lemma, the set $T$ is a $t$-set (note that $U=\{e\}$ in this case, hence $T g \cap T$ and $g T \cap T$ are finite if $g \neq e$ ) while each of the sets $T_{\eta}$ contains large squares. Therefore, $T$ is a $\mathcal{W} \mathcal{A P}(G / N)$-interpolation set by Lemma 3.2 , while none of the sets $T_{\eta}$ is a $\mathcal{B}(G / N)(G)$-interpolation set by Theorem 3.5. 
By Theorem 2.11 there is an isometric embedding

$$
\ell_{\infty}(\kappa) \rightarrow \mathcal{W} \mathcal{A P}(G / N) / \mathcal{B}(G / N) .
$$

Lemma 5.5 then provides the desired copy of $\ell_{\infty}(\kappa)$ in $\mathcal{W} \mathcal{A P}(G) / \mathcal{B}(G)$.

Theorem 5.6 leads naturally also to an improvement of [13, Theorem 4.6].

Theorem 5.7. Let $G$ be a a non-compact, locally compact, nilpotent group and put $\kappa=\kappa(G)$. Then the quotient $\mathcal{W} \mathcal{A P}(G) / \mathcal{B}(G)$ contains a linear isometric copy of $\ell_{\infty}(\kappa)$.

Proof. The case $\kappa(Z(G))=\kappa(G)$ is already proved in Theorem 5.4. So we may assume that $\kappa(Z(G))<\kappa(G)$. We argue by induction on the length $n$ of the upper central series of $G$ (the nilpotency length of $G$ )

$\{e\}=G_{0} \subset G_{1} \subset \ldots \subset G_{n-1} \subset G_{n}=G$ with $Z_{i+1}(G) / Z_{i}(G)=Z\left(G / Z_{i}(G)\right)$.

If $n=1$, then $G$ is Abelian and so Theorem 5.4 or Theorem 5.6 applies.

Assume as inductive hypothesis that the claim holds for groups of nilpotency length at most $n-1$ and suppose $G$ has nilpotency length $n$. Since $\kappa(G)=\kappa(Z(G))+\kappa(G / Z(G))$ and the case $\kappa(Z(G))=\kappa(G)$ has already been ruled out, we can assume that $\kappa(G / Z(G))=\kappa(G)$. Our inductive hypothesis $(\kappa(G / Z(G))$ has nilpotency length $n-1)$ and Lemma 5.5 then provide the desired isometry.

When $G$ is an $I N$-group or a nilpotent group, we recover and improve further the results obtained in Section 4.

Corollary 5.8. Let $G$ be a non-compact IN-group or a nilpotent group and let $\kappa$ be the compact covering of $G$. Then each of the quotient spaces $\mathcal{W} \mathcal{A P}(G) /\left(\mathcal{A P}(G) \oplus C_{0}(G)\right)$ and $\mathcal{W} \mathcal{A} \mathcal{P}_{0}(G) / \mathcal{B}_{0}(G)$ contains a linear isometric copy of $\ell_{\infty}(\kappa)$.

Proof. That the first quotient contains a copy of $\ell_{\infty}(\kappa)$ follows directly from Theorem 5.6 and Theorem 5.7 if we recall the inclusion $\mathcal{A P}(G) \oplus C_{0}(G) \subseteq$ $\mathcal{B}(G)$ (see [13, page 143]).

For the second quotient, we argue as in Theorem 5.6. None of the sets $T_{\eta}, \eta<\kappa$, constructed in all cases needed in the proof of Theorem 5.6, is a $\mathcal{B}_{0}(G)$-interpolation set. On the other hand, proceeding precisely as in Theorems 5.6 and 5.7 (and using the right statements of Lemma 5.5), we see that $T=\bigcup_{\eta<\kappa} T_{\eta}$ is an approximable $\mathcal{W A P}_{0}(G)$-interpolation set.

\section{On the Quotient of $\mathcal{C} \mathcal{B}(G)$ By $\mathcal{L} U \mathcal{C}(G)$}

When $G$ is non-compact, non-discrete, locally compact group, Dzinotyiweyi showed in $[20]$ that the quotient $\mathcal{C} \mathcal{B}(G) / \mathcal{L U C}(G)$ is non-separable. When $G$ is a non-precompact, topological group which is not a P-group, this theorem was generalized and improved in [6, Theorem 3.1] and [7, Theorem 4.1], where a linear isometric copy of $\ell_{\infty}$ was proved to be contained in $\mathcal{C} \mathcal{B}(G) / \mathcal{L U C}(G)$. This section is concerned again with locally compact groups. Our theorem is then more precise and definite. We prove, there is a linear isometric copy of $\ell_{\infty}(\kappa)$ in $\mathcal{C B}(G) / \mathcal{L U C}(G)$, where as before $\kappa$ is the compact covering $G$, if and only if $G$ is neither compact nor discrete. 
Lemma 6.1. Every non-discrete locally compact group contains a faithfully indexed sequence $\left\{x_{n}: n \in \mathbb{N}\right\}$ that converges to the identity.

Proof. A locally compact group always contains a compact subgroup $K$ such that $G / K$ is a metrizable topological space (see [2, Theorem 4.3.29], for instance). Infinite compact groups on the other hand always contain non-trivial convergent sequences ([2, Theorem 4.1.7 and Exercise 4.1.f]). If $K$ is infinite we are done. If $K$ is finite, $G$ is non-discrete and metrizable, it therefore contains non-trivial convergent sequences.

Theorem 6.2. Let $G$ be a locally compact group. Then $\mathcal{C} \mathcal{B}(G) / \mathcal{L U C}(G)$ contains a linear isometric copy of $\ell_{\infty}(\kappa(G))$ if and only if $G$ is neither compact nor discrete.

Proof. The necessity is clear since $\mathcal{C B}(G)=\mathcal{L U C}(G)$ if $G$ is either compact or discrete.

If $G$ is not compact we can find a compact neighbourhood of the identity $U$ and a $U^{2}$-right uniformly discrete subset $X=\left\{x_{\alpha}: \alpha<\kappa\right\} \subseteq G$ with $\kappa=\kappa(G)$. This is clear if $G$ is $\sigma$-compact. If $\kappa>\omega$, we consider $H=$ $\langle U\rangle$, the subgroup generated by $U$. Then $\kappa=|G: H|$ and any system of representatives of right cosets of $H$ constitutes an $H$-right uniformly discrete set of cardinality $\kappa$.

Partition $X$ in $\kappa$-many countable subsets $X=\bigcup_{\alpha<\kappa} X_{\alpha}$. Enumerate, for each $\alpha<\kappa, X_{\alpha}=\left\{x_{\alpha, n}: n<\omega\right\}$. Since $G$ is not discrete, $U$ contains (by Lemma 6.1) a faithfully indexed sequence $S=\left\{s_{n}: n<\omega\right\}$ converging to the identity. With these ingredients, we define

$$
T_{\alpha, n}=\left\{s_{j} x_{\alpha, n}: 1 \leq j \leq n\right\}, T_{\alpha}=\bigcup_{n} T_{\alpha, n} \text { and } T=\bigcup_{\alpha} T_{\alpha} .
$$

Obviously, $U T_{\alpha} \cap U T_{\alpha^{\prime}}=\emptyset$ for every $\alpha \neq \alpha^{\prime}<\kappa$.

Each set $T_{\alpha}$ fails to be an $\mathcal{L U C}(G)$-interpolation set. Indeed, the function $f: T_{\alpha} \rightarrow \mathbb{C}$ such that

$$
\begin{gathered}
f\left(s_{2 j} x_{\alpha, n}\right)=1 \quad \text { for every } \quad j, n \in \mathbb{N} \quad \text { with } \quad 1 \leq 2 j \leq n \text { and } \\
f\left(s_{2 j+1} x_{\alpha, n}\right)=-1 \quad \text { for every } \quad j, n \in \mathbb{N} \quad \text { with } \quad 1 \leq 2 j+1 \leq n
\end{gathered}
$$

cannot coincide on $T_{\alpha}$ with any $\phi \in \mathcal{L U} \mathcal{U}(G)$, since given $\varepsilon>0$, we can choose $j$ large enough and $n \geq 2 j+1$, so that

$$
\left|\phi\left(s_{2 j} x_{\alpha, n}\right)-\phi\left(s_{2 j+1} x_{\alpha, n}\right)\right|<\varepsilon \quad \text { while } \quad f\left(s_{2 j} x_{\alpha, n}\right)-f\left(s_{2 j+1} x_{\alpha, n}\right)=2 .
$$

We now prove that $T$ is an approximable $\mathcal{C} \mathcal{B}(G)$-interpolation set. Since the sequence $\left(s_{j}\right)$ is taken in $U$ and $X$ is right $U^{2}$-uniformly discrete, we readily see that the open set $U x_{\alpha, n} \cap T$ is contained in $\left\{s_{j} x_{\alpha, n}: 1 \leq j \leq n\right\}$. $T$ therefore discrete.

Next we check that $T$ is closed. Since $\lim _{j \rightarrow \infty} s_{j}=e$ and $s_{j} \in U$ for all $j$, there exists a neighbourhood $W$ of the identity such that $W s_{j} \subset U$ for all $j$. Take now a neighbourhood $W_{1}$ of the identity such that $W_{1} W_{1}^{-1} \subseteq W$. Let $x \in G$ be arbitrarily chosen. If, for some $\alpha<\kappa$ and $n<\omega, s_{j, n} x_{\alpha, n} \in W_{1} x$, then $W_{1} x \subseteq U x_{\alpha, n}$. If follows that $W_{1} x \cap T \subseteq U x_{\alpha, n} \cap T$. Since the latter set, see the preceding paragraph, is finite, we conclude that for every $x \in G$, $W_{1} x \cap T$ is either empty or finite. So $T$ is closed. Since the topological space 
underlying $G$ is normal, $T$ is an approximable $\mathcal{C} \mathcal{B}(G)$-interpolation set by Lemma 3.2.

Corollary 2.12 now implies that $\mathcal{e B}(G) / \mathcal{L U}(G)$ contains a linear isometric copy of $\ell_{\infty}(\kappa)$ with $\kappa=|X|=\kappa(G)$.

The equivalence of the first two statements of the following Corollary were proved by Baker and Butcher in [3], see also [28] for a different proof.

Corollary 6.3. Let $G$ be a locally compact group with a compact covering number $\kappa$. Then the following statements are equivalent.

(1) $G$ is neither compact nor discrete.

(2) $\mathcal{e B}(G) \neq \operatorname{LUC}(G)$.

(3) $\mathcal{e B}(G) / \mathcal{L U C}(G)$ contains a linear isometric copy of $\ell_{\infty}(\kappa(G))$.

Proof. (1) $\Longrightarrow(3)$ is proved in the theorem above. (3) $\Longrightarrow(2)$ is obvious and $(2) \Longrightarrow(1)$ is clear.

Remark 6.4. Theorem 6.2 implies a fortiori that the space $L^{\infty}(G) / \mathcal{L U C}(G)$ as well as $L^{\infty}(G) / \mathcal{W} \mathcal{A P}(G)$ contains a linear isometric copy of $\ell_{\infty}(\kappa)$. The arguments used in [6, Section 4], may be applied again to deduce that the group algebra $L^{1}(G)$ is extremely non-Arens regular whenever $\kappa$ is greater or equal to the local character $\chi(G)$ of $G$ (this is the least cardinality of an open base at the identity of $G$.) To obtain the full result, however, harder work is necessary. This is achieved in our recent article [25].

ACKnowledgement. This paper was written when the first author was visiting University of Jaume I in Castellón in December 2010-January 2011. He would like to thank Jorge Galindo for his hospitality and all the folks at the department of mathematics in Castellón. The work was partially supported by Grant INV-2010-20 of the 2010 Program for Visiting Researchers of University Jaume I. This support is also gratefully acknowledged.

\section{REFERENCES}

[1] O. T. Alas, Topological groups and uniform continuity, Portugal. Math. 30 (1971) $137-143$.

[2] A. V. Arhangel'skii and M. G. Tkachenko, Topological Groups and Related Structures, Atlantis Series in Mathematics, vol. I, Atlantis Press/World Scientific, ParisAmsterdam (2008).

[3] J. W. Baker and R. J. Butcher. The Stone-Čech compactification of a topological semigroup. Math. Proc. Camb. Phil. Soc, 80 (1976) 103-107.

[4] J. W. Baker and M. Filali, On the analogue of Veech's theorem in the WAPcompactification of a locally compact group, Semigroup Forum 65 no. 1 (2002) 107112.

[5] J. F. Berglund, H. D. Junghenn and P. Milnes, Analysis on Semigroups: Function Spaces, Compactifications, Representations, Wiley, New York (1989).

[6] A. Bouziad and M. Filali, On the size of quotients of function spaces on a topological group, Studia Math. 202 (2011) 243-259.

[7] A. Bouziad and M. Filali, The Stone-Čech compactification of a topological group as a semigroup and the SIN property, Houston J. Math. 38 no. 4 (2012) 13291341. to appear.

[8] T. Budak, N. Isik and J. Pym, Minimal determinants of topological centres for some algebras associated with locally compact groups, Bull. Lond. Math. Soc. 43 no. 3 (2011) $495-506$. 
[9] R. B. Burckel, Weakly almost periodic functions on semigroups, Gordon and Breach Science Publishers, New York-London-Paris 1970

[10] C. Chou, Weakly almost periodic functions and almost convergent functions on a group, Trans. Amer. Math. Soc. 206 (1975) 175-200.

[11] C. Chou, Uniform closures of Fourier-Stieltjes algebras, Proc. Amer. Math. Soc. 77 (1979) 99-102.

[12] C. Chou, Minimally almost periodic groups. J. Funct. Anal. 36 (1980) 1-17.

[13] C. Chou, Weakly almost periodic functions and Fourier-Stieltjes algebras of locally compact groups, Trans. Amer. Math. Soc., 274 no. 1 (1982) 141-157.

[14] C. Chou, Weakly almost periodic functions and thin sets in discrete groups. Trans. Amer. Math. Soc. 321 no. 1 (1990) 333-346.

[15] P. Civin and B. Yood, The second conjugate space of a Banach algebra as an algebra, Pacific J. Math. 11 (1961), 847-870.

[16] W. Comfort and K. Ross, Pseudocompactness and uniform continuity in topological groups, Pacific J. Math. 16 (1966) 483-496.

[17] H. G. Dales and, A. T.-M. Lau, The second duals of Beurling algebras, Mem. Amer. Math. Soc. 177 (2005).

[18] S. W. Drury. Sur les ensembles de Sidon. C. R. Acad. Sci. Paris Sér. A-B 271 (1970) A162-A163.

[19] C. F. Dunkl and D. E. Ramirez. Topics in harmonic analysis. Appleton-CenturyCrofts [Meredith Corporation], New York, (1971). Appleton-Century Mathematics Series.

[20] A. M. H. Dzinotyiweyi, Nonseparability of quotient spaces of function algebras on topological semigroups, Trans. Amer. Math. Soc. 272 (1982) 223-235.

[21] R. Engelking. General topology. PWN-Polish Scientific Publishers, Warsaw, (1977).

[22] P. Eymard, L'algèbre de Fourier d'un groupe locallement compact, Bull. Soc. Math. France 92 (1964) 181-236.

[23] M. Filali, On the actions of a locally compact group on some of its semigroup compactifications, Math. Proc. Cambridge Philos. Soc. 143 (2007) 25-39.

[24] M. Filali and J. Galindo, Approximable $\mathcal{W} \mathcal{A P}$ - and $\mathcal{L} \mathcal{U}(G)$-interpolation sets, Advances in Math. 233 (2013) 87-114.

[25] M. Filali and J. Galindo, Extreme non-Arens regularity of the group algebra, Preprint (2013).

[26] M. Filali and J. S. Pym, Right cancellation in the LUC-compactification of a locally compact group, Bull. London Math. Soc. 35 (2003) 128-134.

[27] M. Filali and P. Salmi, Slowly oscillating functions in semigroup compactification and convolution algebras, Journal of Functional Analysis 250 no. 1 (2007) 144-166.

[28] M. Filali and T. Vedenjuoksu, The Stone-Čech compactification of a topological group and the $\beta$-extension property, Houston J. Math. 36 no. 2 (2010) 477-488.

[29] J. Galindo, On group and semigroup compactifications of topological groups. Manuscript in preparation.

[30] C. C. Graham and K. E. Hare, Interpolation and Sidon sets for compact groups, CMS Books in Mathematics/Ouvrages de Mathématiques de la SMC. Springer, New York, (2013).

[31] E. E. Granirer, Exposed points of convex sets and weak sequential convergence, Memoirs of the American Mathematical Society, No. 123. American Mathematical Society, Providence, R.I., (1972).

[32] E. E. Granirer, The radical of $L^{\infty}(G)^{*}$, Proc. Amer. Math. Soc. 41 (1973) 321-324.

[33] S. Grosser, and M. Moskowitz, Compactness conditions in topological groups, J. Reine Angew. Math. 246 (1971) 1-40.

[34] S. L. Gulick, Commutativity and ideals in the biduals of topological algebras, Pacific J. Math. 18 (1966), 121-137.

[35] E. Hewitt and K. A. Ross, Abstract harmonic analysis I, Springer-Verlag, Berlin, (1963).

[36] N. Hindman and D. Strauss, Algebra in the Stone-Čech Compactification, de Gruyter Exp. Math. 27, Walter de Gruyter, Berlin, (1998). 
[37] T. Jech, Set Theory, Springer, The third millennium edition, revised and expanded, Berlin (2006).

[38] A. T. -M. Lau, Continuity of Arens multiplication on the dual space of bounded uniformly continuous functions on locally compact groups and topological semigroups, Math. Proc. Cambridge Philos. Soc. 99 (1986) 273-283.

[39] A. T. M. Lau and V. Losert, On the second conjugate of $L^{1}(G)$ of a locally compact group, J. London Math. Soc. 37 (1988) 464-470.

[40] A. T. M. Lau, J. S. Pym, The topological centre of a compactification of a locally compact group, Math. Z. 219 no. 4 (1995) 567-579.

[41] A. T. M. Lau, A. Ülger, Topological centers of certain dual algebras, Trans. Amer. Math. Soc. 348 (1996) 1191-1212.

[42] K. de Leeuw and I. Glicksberg, Almost periodic functions on semigroups, Acta Math. 105 (1961) 99-140.

[43] K. de Leeuw and I. Glicksberg, The decomposition of certain group representations Analyse Math. 15 (1965) 135-192.

[44] M. Neufang, A unified approach to the topological centre problem for certain Banach algebras arising in abstract harmonic analysis, Arch. Math. (Basel) 82 no. 2 (2004) 164-171.

[45] B. H. Neumann, Groups covered by finitely many cosets. Publ. Math. Debrecen (1954) 227-242 (1955).

[46] J. Pym, A note on $G^{\mathcal{L U C}}$ and Veech's theorem, Semigroup Forum 59 (1999) 171-174.

[47] D. E. Ramirez, Weakly almost periodic functions and Fourier-Stieltjes transforms, Proc. Amer. Math. Soc. 19 (1968) 1087-1088.

[48] H. P. Rosenthal, On injective Banach spaces and the spaces $L^{\infty}(\mu)$ for finite measures $\mu$, Acta Math. 124 (1970) 205-248.

[49] W. Rudin, Weak almost periodic functions and Fourier-Stieltjes transforms, Duke Math. J. 26 (1959) 215-220.

[50] W. Ruppert, On weakly almost periodic sets, Semigroup Forum 32 (1985) 267-281.

[51] W. Ruppert, Compact semitopological semigroups: an intrinsic theory, Lecture Notes in Mathematics, 1079. Springer-Verlag, Berlin, 1984.

[52] W. A. Veech. Topological dynamics. Bull. Amer. Math. Soc. 83 (1977) 775-830.

[53] W. A. Veech, Weakly almost periodic functions on semisimple Lie groups, Monatsh. Math. 88 no. 1 (1979) 55-68.

[54] N. J. Young, The irregularity of multiplication in group algebras, Quart J. Math. Oxford Ser. 24 (1973) 59-62.

Mahmoud Filali, Department of Mathematical Sciences, University of Oulu, Oulu, Finland.

E-MAIL: mfilali@cc.oulu.fi

Jorge Galindo, Instituto Universitario de Matemáticas y Aplicaciones (IMAC),, Universidad Jaume I, E-12071, Castellón, Spain.

E-MAIL: jgalindo@mat.uji.es 